\title{
MARKOV PROCESSES WHOSE HITTING DISTRIBUTIONS ARE DOMINATED BY THOSE OF A GIVEN PROCESS $\left({ }^{1}\right)$
}

\author{
BY \\ CHUNG-TUO SHIH
}

Introduction. Let $X$ be a Markov process. Let $X^{*}$ be the process obtained from a random time change in a subprocess of $X$. Then obviously the hitting distributions of $X^{*}$ are dominated by those of $X$. It has been naturally conjectured that the converse is true under broad conditions. The exact statement of the converse would be as follows: if two Markov processes are such that the hitting distributions of $X$ dominate those of $X^{*}$, then there is a process $Y$ obtained from a random time change in a subprocess of $X$ that is equivalent to $X^{*}$. The conjecture is proved by Sur [6] in case $X$ is a Brownian motion process and $X^{*}$ is a standard process. This paper deals with the very general case where $X$ and $X^{*}$ are Hunt processes with a locally compact separable metric space as their common state space. (Our definition of a Hunt process agrees essentially with that of a standard process.) The conjecture is proved true under a trivially necessary condition, provided a slight change in the definition of a subprocess is allowed (see $\$ 1$ ). Our method is to construct a multiplicative functional of $X$ which induces a process with the same hitting distributions as $X^{*}$. When the state space of $X^{*}$ is a proper subspace of that of $X$ which is a locally compact separable metric space, it seems that no difficulty would arise in obtaining a similar result with the same techniques.

1. Preliminaries and the main result. Let $E$ be a locally compact separable metric space. Let $E_{\Delta}=E \cup\{\Delta\}$ be the one-point compactification of $E$. Denote by $\mathscr{B}$ and $\mathscr{B}_{\Delta}$ respectively the Borel fields of $E$ and $E_{\Delta}$. A Hunt process $X$ with $E$ as its state space is a structure $\left(\Omega, \mathscr{M}, X(t), P^{x}, \theta_{t}\right)$ where $\Omega$ is a set (the sample space), $\mathscr{M}$ a $\sigma$-field on $\Omega, X(t), 0 \leqq t<\infty$, are random variables on $(\Omega, \mathscr{M})$ taking values in $E_{\Delta}, P^{x}, x \in E_{\Delta}$, probability measures on $\mathscr{M}$ with $P^{x}(X(0)=x)=1$, and $\theta_{t}$, $0 \leqq t<\infty$, operators on $\Omega$ satisfying $X(s)\left(\theta_{t}\right)=X(s+t)$. For each $\omega \in \Omega$, the path function $t \rightarrow X_{t}(\omega)=X(t, \omega)=X(t)(\omega)$ is right continuous and has a left limit at every $t$, and $X(t, \omega)=\Delta$ if $t \geqq \sigma(\omega)=\inf \{s \mid X(s)=\Delta\}$, the lifetime of $\omega$. Let $\mathscr{G}_{t}$ be the $\sigma$-field generated by $X(s), s \leqq t$ and $\mathscr{G}$ that generated by $X(t), t<\infty$. For each

Presented to the Society, October 30, 1965; received by the editors September 1, 1966.

(1) This is essentially the author's doctoral dissertation at the University of Washington written under the supervision of Professor R. M. Blumenthal, to whom the author wishes to express his deep gratitude for encouragement and advice. Some improvement has been made while the author held an ONR Research Associateship in Mathematics at Cornell University. 
$\Lambda \in \mathscr{G}, x \rightarrow P^{x}(\Lambda)$ is $\mathscr{B}_{\Delta}$-measurable. For a probability measure $\mu$ on $\mathscr{B}_{\Delta}(\mu$ will always denote such a measure) $P^{\mu}$ is defined on $\mathscr{G}$ by $P^{\mu}(\Lambda)=\int P^{x}(\Lambda) \mu(d x)$. Let $\mathscr{G}^{\mu}$ be the $P^{\mu}$-completion of $\mathscr{G}$, and let $\mathscr{F}$ be the intersection of $\mathscr{G}^{\mu}$ over all $\mu$. Let $\mathscr{F}_{t}$ be the $\sigma$-field of sets $\Lambda$ satisfying the condition that, for each $\mu$, there exist $\Lambda_{1} \in \mathscr{G}_{t}$ and $\Lambda_{2} \in \mathscr{G}$ with $P^{\mu}\left(\Lambda_{2}\right)=0$ and $\left(\Lambda-\Lambda_{1}\right) \cup\left(\Lambda_{1}-\Lambda\right) \subset \Lambda_{2}$. A stopping time $T$ is a function from $\Omega$ to $[0, \infty]$ such that $\{T<t\} \in \mathscr{F}_{t}$ for every $t$. For a stopping time $T$, $\mathscr{F}_{T}=\mathscr{F}(T)$ denotes the $\sigma$-field of sets $\Lambda \in \mathscr{F}$ satisfying $\Lambda \cap\{T<t\} \in \mathscr{F}_{t}$ for every $t$. If $\phi$ is a bounded real-valued $\mathscr{F}$-measurable function and $T$ a stopping time, then $E^{x}\left\{\phi\left(\theta_{T}\right) ; \Lambda \cap[T<\infty]\right\}=E^{x}\left\{E^{X(T)}(\phi) ; \Lambda \cap[T<\infty]\right\}$ for all $\Lambda \in \mathscr{F}(T)$. This is the strong Markov property of $X$. If $T_{n}$ are stopping times increasing to $T$, then $X\left(T_{n}\right)$ converge to $X(T)$ a.e. on $\{T<\sigma\}$, where a.e. means a.e. $P^{x}$ for every $x$. This is called the quasi-left continuity of $X$. Note that it is not required that $X$ satisfies the stronger form of quasi-left continuity that the above convergence holds a.e. on $\{T<\infty\}$. Thus our definition of a Hunt process agrees with that of a standard process.

For an analytic subset $A$ of $E$ and $x \in E$, the hitting distribution $H_{A}(x, \cdot)$ is defined on $\mathscr{B}$ by $H_{A}(x, B)=P^{x}\left(X\left(T_{A}\right) \in B, T_{A}<\infty\right)$, where $T_{A}=\inf \{t>0 \mid X(t) \in A\}$ is the hitting time of $A$. A point $x$ is regular for $A$ if $P^{x}\left(T_{A}=0\right)=1$, and irregular if otherwise. A point $x \in E$ is a holding point if it is irregular for $E-\{x\}$. The set of holding points will be denoted by $H$. The points in $I=E-H$ are called instantaneous points. A holding point is called a trap if $H_{E-\{x\}}(x, E)=0$. Let $T$ be a stopping time $\leqq \sigma$ such that $T\left(\theta_{t}\right)=T-t$ on $\{t<T\}$. Let $Z(t)=X(t)$ on $\{t<T\}$ and $=\Delta$ elsewhere. Then $Z=\left(\Omega, \mathscr{M}, Z(t), P^{x}, \theta_{t}\right)$ is again a Hunt process, whose state space is $E^{\prime}=\left\{x \in E \mid P^{x}(T>0)=1\right\}$. (If $X$ is only a strong Markov process then so is $Z$.) Such a process is called a subprocess of $X$. A multiplicative functional $M$ of $X$ is a real-valued function on $[0, \infty) \times \Omega$ such that (i) $t \rightarrow M(t)(\omega)=M(t, \omega)$ is right continuous, nonincreasing and taking values in $[0,1]$ for almost all $\omega$, (ii) $M(t)$ is $\mathscr{F}(t)$-measurable, and (iii) $M(s+t)=M(t)\left[M(s)\left(\theta_{t}\right)\right]$ a.e. If $M$ is a multiplicative functional such that $M(t)=0$ on $\{\sigma \leqq t\}$, then one can define a Markov process $Y$ in the manner of [5, p. 142] from $X$ and $M . Y$ has $E^{\prime}=\left\{x \in E \mid P^{x}(M(0)=1)=1\right\}$ as its state space. The transition function $q(t, x, B), x \in E^{\prime}, B \in \mathscr{B} \cap E^{\prime}$, of $Y$ is given by $E^{x}\{M(t) ; X(t) \in B\}$ and its hitting distribution $H_{A}^{X}(x, B), x \in E, A \subset E^{\prime}$, is equal to $E^{x}\left\{M\left(T_{A^{\prime}}\right) ; X\left(T_{A^{\prime}}\right) \in B, T_{A^{\prime}}<\infty\right\}$ where $A^{\prime}=\left(E-E^{\prime}\right) \cup A$. Such a process we will also call a subprocess of $X$.

For a summary of the basic definitions and facts of the theory of Hunt processes we refer the reader to $\S 1$ of [4] or $\S 2$ of [1]. A discussion of multiplicative functionals can be found in [3], [6] or Chapter 10 of [2].

Let us consider two Hunt processes $X$ and $X^{*}$ with $E$ as their common state space. For notational convenience suppose $X$ and $X^{*}$ are defined on the same sample space $(\Omega, \mathscr{M})$ and by the same random variables $X(t)$. Thus we write $X^{*}=\left(\Omega, \mathscr{M}, X(t),{ }^{*} P^{x}, \theta_{t}\right)$. Let ${ }^{*} P^{\mu}, \mathscr{F}^{*}, \mathscr{F}_{t}^{*}, \mathscr{F}_{T}^{*}, H_{A}^{*}(x, B), H^{*}$ and $I^{*}$ be defined from the probability measures ${ }^{*} P^{x}$ in the same manner as $P^{\mu}, \mathscr{F}$, etc. are defined 
from $P^{x}$; a.e. ${ }^{*}$ will mean a.e. ${ }^{*} P^{x}$ for every $x$. If $\mathscr{S}$ is sub- $\sigma$-field of $\mathscr{F}(\mathscr{F} *)$, $\mathscr{S}^{\mu}\left(\mathscr{S}^{* \mu}\right)$ will denote the $\sigma$-field of sets $\Lambda$ such that there exist $\Lambda_{1} \in \mathscr{S}$ and $\Lambda_{2} \in \mathscr{F}\left(\Lambda_{2} \in \mathscr{F} *\right)$ with $P^{\mu}\left(\Lambda_{2}\right)=0\left({ }^{*} P^{\mu}\left(\Lambda_{2}\right)=0\right)$ and $\left(\Lambda-\Lambda_{1}\right) \cup\left(\Lambda_{1}-\Lambda\right) \subset \Lambda_{2}$. Note that this definition agrees with a previous one if $\mathscr{S}=\mathscr{G}$. For $\Lambda \subset \Omega, \mathscr{S} \cap \Lambda$ $=\left\{\Lambda_{1} \cap \Lambda \mid \Lambda_{1} \in \mathscr{S}\right\}$. Let $\mathscr{C}_{0}$ be the set of real continuous functions on $E$ vanishing at infinity.

Our basic hypotheses are:

A. $H_{K}^{*}(x, \cdot) \leqq H_{K}(x, \cdot)$ for every compact subset $K$ of $E$ and $x \in E$.

B. If $x$ is a trap for $X^{*}$, then $x \in H$.

The main result is the following

THEOREM. Under hypotheses A and B there exists a multiplicative functional $M$ of $X$ such that $E^{x}\left\{M\left(T_{A}\right) ; X\left(T_{A}\right) \in B, T_{A}<\infty\right\}=H_{A}^{*}(x, B)$ for all $x \in E$, analytic subsets $A$ of $E$ and $B \in \mathscr{B}$.

Obviously the multiplicative functional $M$ in the theorem must satisfy $M(0)=1$ a.e. $P^{x}$ for all $x \in E$, and we may assume $M(t)=0$ on $\{\sigma \leqq t\}$. Thus the subprocess $Y$ of $X$ induced by $M$ has $E$ as its state space and has the same hitting distributions (on analytic subsets of $E$ ) as $X^{*}$. One can then appeal to the result of [1] to obtain a random time change in $Y$ which induces a process equivalent to $X^{*}$.

A remark about the hypotheses: if the result of the theorem holds and if $x \in I$, then $x$ is not a trap for $X^{*}$, since

$$
H_{E-\{x\}}^{*}(x, E)=E^{x}\left\{M\left(T_{E-\{x\}}\right) ; X\left(T_{E-\{x\}}\right) \in E, T_{E-\{x\}}<\infty\right\}=1 .
$$

Thus hypothesis B is necessary. Hypothesis A implies that $H_{A}^{*}(x, \cdot) \leqq H_{A}(x, \cdot)$ for all analytic subsets $A$ of $E$. This is a result of the fact that, in a Hunt process, the hitting time of an analytic set can be approximated by the hitting time of its compact subsets.

Let us choose a metric $\rho$ on $E_{\Delta}$. Let $\operatorname{reg} A\left(\operatorname{reg}^{*} A\right)$ be the set of regular points for $A$ relative to $X\left(X^{*}\right)$. We will now prove two immediate results of hypotheses $\mathrm{A}$ and $\mathrm{B}$.

Proposition 1.1. $H=H^{*}$.

Proof. If $x \in H$, then $H_{E-\{x\}}^{*}(x,\{x\}) \leqq H_{E-\{x\}}(x,\{x\})=0$, and hence $x$ cannot be in $I^{*}$. So $H \subset H^{*}$. Suppose $x \in H^{*}$. If $H_{E-\{x\}}^{*}(x, E-\{x\})=0$, then $x$ is a trap for $X^{*}$ and so $x \in H$ by hypothesis B. If $H_{E-\{x\}}^{*}(x, E-\{x\})>0$, then $H_{E-\{x\}}(x, E-\{x\})>0$ and so $x \notin I$. So $H^{*} \subset H$.

Proposition 1.2. If $K$ is a compact subset of $E$, then reg $K=\operatorname{reg}^{*} K$.

Proof. If $x \in K$, then trivially $x \notin \operatorname{reg} K$ and also $x \notin \operatorname{reg}^{*} K$. Suppose $x \in K$. If $x \in H=H^{*}$, then of course $x \in \operatorname{reg} K \cap \operatorname{reg}^{*} K$. Suppose $x \in I=I^{*}$. Let

$$
D_{n}=K \cup\{y \mid 1 / n \leqq \rho(x, y) \leqq \rho(x, \Delta) / 2\} .
$$


Then we clearly have: $H_{D_{n}}(x,\{x\})=1$ for all $n$ if and only if $x \in \operatorname{reg} K, H_{D_{n}}^{*}(x,\{x\})=1$ for all $n$ if and only if $x \in$ reg* $K$ and $H_{D_{n}}^{*}\left(x, D_{n}\right) \uparrow 1$ as $n \rightarrow \infty$. These conditions and hypothesis $\mathrm{A}$ imply that $x \in \operatorname{reg} K$ if and only if $x \in \mathrm{reg}^{*} K$. The proof is complete.

2. Partitioning of the state space and a family of stopping times. A pair $(\mathscr{U}, \mathscr{V})$ will be called a partition of $E$ if $\mathscr{U}=\left\{U_{1}, \ldots, U_{p}\right\}$ is a finite covering of $E$ by open subsets of $E$, and $\mathscr{V}=\left\{V_{1}, \ldots, V_{p}\right\}$ is an (ordinary) partition of $E$ by Borel sets with $V_{i} \subset U_{i}$ for all $i$. A partition $(\mathscr{U}, \mathscr{V})$ of $E$ is a refinement of another partition $\left(\mathscr{U}^{\prime}, \mathscr{V}^{\prime}\right)$ if, whenever $V_{i} \in \mathscr{V}, U_{j}^{\prime} \in \mathscr{U}^{\prime}$, and $V_{i} \cap U_{j}^{\prime} \neq \varnothing$, we have $U_{i} \subset U_{j}^{\prime}$. It is easily checked that being a refinement of is a transitive relation among partitions $(\mathscr{U}, \mathscr{V})$ of $E$. For a family $\mathscr{U}$ of subsets of $E$ let $|\mathscr{U}|$ be the sup over $U \in \mathscr{U}$ of diam $U=\sup \{\rho(x, y) \mid x, y \in U\}$. The following observation is basic to our study.

Proposition 2.1. There exists a sequence $\left(\mathscr{U}^{(n)}, \mathscr{V}^{(n)}\right), n=1,2, \ldots$, of partitions of $E$ such that (i) $\left|\mathscr{U}^{(n)}\right|<1 / n$ for all $n$, (ii) $\left(\mathscr{U}^{(n)}, \mathscr{V}^{(n)}\right)$ is a refinement of $\left(\mathscr{U}^{(m)}, \mathscr{V}^{(m)}\right)$ if $m \leqq n$, and (iii) $V_{i}^{(n)}=U_{i}^{(n)}-\bigcup_{j=1}^{i-1} U_{j}^{(n)}$ for all $n$ and $i>1$.

Proof. For each $n$ choose a finite covering $\mathscr{W}^{(n)}$ of $E$ consisting of open subsets of $E$ such that $\left|\mathscr{W}^{(n)}\right|<1 / n$ (note that $\rho$ is a metric on $E_{\Delta}$ ) and $\mathscr{W}^{(n)}$ is closed w.r.t. finite intersection. Define $\mathscr{U}^{(n)}$ inductively by setting $\mathscr{U}^{(1)}=\mathscr{W}^{(1)}$ and $\mathscr{U}^{(n+1)}$ $=\left\{U_{1} \cap U_{2} \mid U_{1} \in \mathscr{U}^{(n)}\right.$ and $\left.U_{2} \in \mathscr{W}^{(n+1)}\right\}$ for $n \geqq 1$. Clearly each $\mathscr{U}^{(n)}$ is closed w.r.t. finite intersection. Enumerate numbers of $\mathscr{U}^{(n)}$ as $U_{1}^{(n)}, \ldots, U_{p}^{(n)}$ in such a manner that $i \leqq j$ whenever $U_{i}^{(n)} \subset U_{j}^{(n)}$. Let $V_{1}^{(n)}=U_{1}^{(n)}$ and $V_{i}^{(n)}=U_{i}^{(n)}-\bigcup_{j=1}^{i-1} U_{j}^{(n)}$ for $i>1$. (i) and (iii) are immediate. We now show $\left(\mathscr{U}^{(n+1)}, \mathscr{V}^{(n+1)}\right)$ refines $\left(\mathscr{U}^{(n)}, \mathscr{V}^{(n)}\right)$. Suppose $x \in V_{i}^{(n+1)} \cap U_{j}^{(n)}$. We need to show $U_{i}^{(n+1)} \subset U_{j}^{(n)}$. It is clear that $U_{i}^{(n+1)}$ $=\bigcap\left\{U \in \mathscr{U}^{(n+1)} \mid x \in U\right\}$. On the other hand $U_{i}^{(n+1)}=U_{1} \cap U_{2}$ for some $U_{1} \in \mathscr{U}^{(n)}$ and $U_{2} \in \mathscr{W}^{(n+1)}$. Since $x \in U_{j}^{(n)} \cap U_{2} \in \mathscr{U}^{(n+1)}$, we have $U_{i}^{(n+1)} \subset U_{j}^{(n)} \cap U_{2} \subset U_{j}^{(n)}$. This proves $\left(\mathscr{U}^{(n+1)}, \mathscr{V}^{(n+1)}\right)$ is a refinement of $\left(\mathscr{U}^{(n)}, \mathscr{V}^{(n)}\right)$, and it follows from transitivity that $\left(\mathscr{U}^{(n)}, \mathscr{V}^{(n)}\right)$ refines $\left(\mathscr{U}^{(m)}, \mathscr{V}^{(m)}\right)$ if $m<n$. It remains to show $\left(\mathscr{U}^{(n)}, \mathscr{V}^{(n)}\right)$ is a refinement of itself. But this is obvious since if $x \in V_{i}^{(n)} \cap U_{j}^{(n)}$, then $U_{i}^{(n)}=$ $\bigcap\left\{U \in \mathscr{U}^{(n)} \mid x \in U\right\} \subset U_{j}^{(n)}$. We choose a sequence $\left\{\left(\mathscr{U}^{(n)}, \mathscr{V}^{(n)}\right)\right\}$ of partitions of $E$ satisfying the conditions in the above proposition. Let us define a family of stopping times as follows. First, for each $n$ let $T^{(n)}=T_{E-U_{i}^{(n)}}$ if $X(0) \in V_{i}^{(n)}$, and $=\infty$ if $X(0)=\Delta$. It is clear that each $T^{(n)}$ is a stopping time (for both processes $X$ and $X^{*}$-as is every stopping time to be defined in the sequel). Let $\pi$ be the first uncountable ordinal. For each $n$ define inductively stopping times $T_{\alpha}^{(n)}, \alpha<\pi$, as follows:

$$
\begin{aligned}
& T_{0}^{(n)}=0 ; T_{\alpha+1}^{(n)}=T_{\alpha}^{(n)}+T^{(n)}\left(\theta_{T_{\alpha}^{(n)}} \quad \text { if } T_{\alpha}^{(n)}<\infty, \text { and }=\infty \text { if } T_{\alpha}^{(n)}=\infty ;\right. \\
& T_{\alpha}^{(n)}=\sup _{\gamma<\alpha} T_{\gamma}^{(n)} \quad \text { if } \alpha \text { is a limit ordinal. }
\end{aligned}
$$

The right continuity of path functions implies that $T^{(n)}>0$ and hence $T_{\alpha+1}^{(n)}>T_{\alpha}^{(n)}$ provided $T_{\alpha}^{(n)}<\infty$. It follows that for each $\omega$ there is $\alpha<\pi$ such that $T_{\alpha}^{(n)}(\omega)=\infty$. Also, a standard argument shows that for each $\mu$ there exists $\beta<\pi$ such that $P^{\mu}\left(T_{\beta}^{(n)}<\infty\right)={ }^{*} P^{\mu}\left(T_{\beta}^{(n)}<\infty\right)=0$. 
Proposition 2.2. Let $m<n$ and $\omega \in \Omega$. If $X(0, \omega) \in U_{i}^{(m)}$, then, for some $\alpha$, $T_{\alpha}^{(n)}(\omega)=T_{E-U_{i}^{(m)}}(\omega)$.

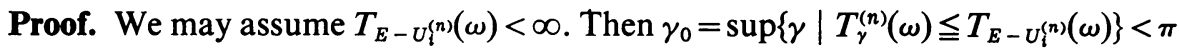
and $T_{\gamma_{0}}^{(n)}(\omega) \leqq T_{E-U_{i}^{(m)}}(\omega)$. If the equality does not hold, then $x=X\left(T_{\gamma_{0}}^{(n)}(\omega), \omega\right) \in U_{i}^{(n)}$.

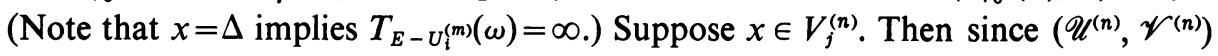
is a refinement of $\left(\mathscr{U}^{(m)}, \mathscr{V}^{(m)}\right)$ we have $U_{j}^{(n)} \subset U_{i}^{(m)}$. This implies $T^{(n)}\left(\theta_{T_{y_{0}}^{(n)}}\right)$ $\leqq T_{E-U_{i}^{(m)}}\left(\theta_{\left.T_{\gamma_{0}}^{(n)} \omega\right)}\right.$ and hence $T_{\gamma_{0}+1}^{(n)}(\omega) \leqq T_{E-U_{i}^{(m)}}(\omega)$, a contradiction.

Corollary 2.3. Let $m<n, \alpha<\pi$, and $\omega \in \Omega$. There is $\lambda<\pi$ satisfying

$$
T_{\lambda}^{(n)}(\omega)=T_{\alpha}^{(m)}(\omega)
$$

Proof. The case $\alpha=1$ follows immediately from the previous proposition and the definition of $T_{1}^{(m)}(\omega)$. Suppose the assertion is true if $\alpha$ is replaced by any smaller ordinal. If $\alpha$ has a predecessor $\gamma$ and $T_{\gamma}^{(m)}(\omega)=T_{\lambda_{1}}^{(n)}(\omega)$, then assuming as we may that $T_{\gamma}^{(m)}(\omega)<\infty$, we can find $\lambda_{2}$ such that $T_{\lambda_{2}}^{(n)}\left(\theta_{\left.T_{\lambda_{1}}^{(n)} \omega\right)}=T_{\lambda_{2}}^{(n)}\left(\theta_{\left.T_{\gamma}^{(m)} \omega\right)}=T_{1}^{(m)}\left(\theta_{\left.T_{y}^{(m)} \omega\right) \text {. Thus }}\right.\right.\right.$ we have $T_{\lambda_{1}+\lambda_{2}}^{(n)}(\omega)=T_{\alpha}^{(m)}(\omega)$. If $\alpha$ is a limit ordinal, let $\lambda(\gamma)$ be such that $T_{\lambda(\gamma)}^{(n)}(\omega)$ $=T_{\gamma}^{(m)}(\omega)$ for $\gamma<\alpha$ and let $\lambda=\sup \{\lambda(\gamma) \mid \gamma<\alpha\}$. Then $T_{\lambda}^{(n)}(\omega)=T_{\alpha}^{(m)}(\omega)$ by definition. The corollary then follows from induction.

The fact asserted in the above corollary, which may be called the interpolation property of the $T_{\alpha}^{(n)}$, is fundamental to this work. Let $\lambda(m, \alpha, n)=\inf \left\{\lambda<\pi \mid T_{\lambda}^{(n)}=T_{\alpha}^{(m)}\right\}$ for $m \leqq n$. Note that $\lambda(m, \alpha, n)$ is a function from $\Omega$ into the set of countable ordinals, and $\{\lambda(m, \alpha, n)=\gamma\}=\left\{T_{\alpha}^{(m)}=T_{\gamma}^{(n)}>T_{\delta}^{(n)}>\right.$ for all $\left.\delta<\gamma\right\}$ is in $\mathscr{F}\left(T_{\alpha}^{(m)}\right) \cap \mathscr{F}\left(T_{\gamma}^{(n)}\right)$ and also in $\mathscr{F}\left(T_{\alpha}^{(m)}\right)^{*} \cap \mathscr{F}\left(T_{\gamma}^{(n)}\right)^{*}$.

Corollary 2.4. Let $m<n$. If $T_{\alpha}^{(m)}(\omega) \geqq t$, there exists $\gamma<\pi$ such that

$$
T_{\alpha}^{(m)}(\omega)=t+T_{\gamma}^{(n)}\left(\theta_{t} \omega\right) \text {. }
$$

Proof. The case $T_{\alpha}^{(m)}(\omega)=t$ is trivial and we may assume $T_{\alpha}^{(m)}(\omega)>t$. Let $\alpha_{0}=\sup \left\{\alpha_{1} \mid T_{\alpha_{1}}^{(m)}(\omega) \leqq t\right\}$. Then $T_{\alpha_{0}}^{(m)}(\omega) \leqq t<T_{\alpha_{0}+1}^{(m)}(\omega)$. Suppose $X\left(T_{\alpha_{0}}^{(m)}(\omega), \omega\right) \in V_{i}^{(m)}$. Then $X(t, \omega) \in U_{i}^{(m)}$ and $T_{\alpha_{0}+1}^{(m)}-t$ is the first time $\theta_{t} \omega$ hits $E-U_{i}^{(m)}$. Now Proposition 2.2 implies that $T_{\alpha_{0}+1}^{(m)}(\omega)-t=T_{\gamma}^{(n)}\left(\theta_{t} \omega\right)$ for some $\gamma$. This proves the case when $\alpha=\alpha_{0}+1$. The case when $\alpha>\alpha_{0}+1$ is then easy to see.

For a compact subset $K$ of $E$, we can define stopping times in terms of $T_{\alpha}^{(n)}$ to approximate $T_{K}$. Let $T_{n}=\inf \left\{T_{\alpha}^{(n)}<\sigma \mid\right.$ if $X\left(T_{\alpha}^{(n)}\right) \in V_{i}^{(n)}$ then $\left.U_{i}^{(n)} \cap K \neq \varnothing\right\}$ and $R_{n}=\inf \left\{T_{\alpha}^{(n)} \mid T_{\alpha}^{(n)} \geqq T_{K}\right\}$.

Proposition 2.5. Each $T_{n}$ is a stopping time. If $x \notin K$, then $T_{n} \uparrow T_{K}$ or $\sigma$ a.e. $P^{x}$ and a.e. ${ }^{*} P^{x}$ as $n \rightarrow \infty$.

Proof. Let $F_{n}=\bigcup\left\{V_{i}^{(n)} \mid U_{i}^{(n)} \cap K \neq \varnothing\right\}$. From the fact that $\left(\mathscr{U}^{(n)}, \mathscr{V}^{(n)}\right)$ refines itself one easily checks that $T_{n}=0$ if $X(0) \in F_{n}$ and $T_{n}=T_{F_{n}}$ if otherwise. Hence $T_{n}$ is a stopping time. The rest follows from the fact that $\rho(y, K)=\inf \{\rho(y, z) \mid z \in K\}$ $<1 / n$ for $y \in F_{n}$ and the quasi-left continuity. 
Proposition 2.6. Each $R_{n}$ is a stopping time. If $K \subset I$, then $R_{n} \downarrow T_{K}$ a.e. and a.e.*.

Proof. $\left\{R_{n}<t\right\}=\bigcup_{\alpha<\pi}\left\{R_{n}=T_{\alpha}^{(n)}<t\right\}$. Now $\left\{R_{n}=T_{\alpha}^{(n)}\right\}=\left\{T_{\alpha}^{(n)} \geqq T_{K}>T_{\gamma}^{(n)}\right.$ for all $\gamma<\alpha\} \in \mathscr{F}\left(T_{\alpha}^{(n)}\right)$. Hence $\left\{R_{n}=T_{\alpha}^{(n)}<t\right\} \in \mathscr{F}(t)$. From the fact that for each $\mu$ there exists $\beta$ such that $P^{\mu}\left(T_{\beta}^{(n)}<\infty\right)=0$ we see that $\left\{R_{n}<t\right\} \in \mathscr{F}(t)$. Similarly $\left\{R_{n}<t\right\} \in \mathscr{F}(t)^{*}$. Hence $R_{n}$ is a stopping time. Suppose $K \subset I=I^{*}$. It follows from the strong Markov property that, a.e. (a.e.*) on $\left\{T_{K}<\infty\right\}$, there is an arbitrarily small $\delta>0$ such that $X\left(T_{K}\right) \neq X\left(T_{K}+\delta\right)$. Clearly if $X(t, \omega) \neq X(t+\delta, \omega)$ then there exists some $(n, \alpha)$ such that $t<T_{\alpha}^{(n)}(\omega) \leqq t+\delta$. Hence $R_{n} \downarrow T_{K}$ a.e. and a.e.*.

3. Some measurability lemmas. For each positive integer $n$ and countable ordinal $\gamma$, let $\mathscr{H}(n, \gamma)$ be the $\sigma$-field generated by sets of the form

$$
\left\{X\left(T_{\delta}^{(n)}\right) \in B, T_{\delta}^{(n)}<\infty\right\}, \quad B \in B_{\Delta}, \delta \leqq \gamma .
$$

Since $\left\{X\left(T_{\delta}^{(n)}\right) \in B, T_{\delta}^{(n)}<\infty\right\} \in \mathscr{F}\left(T_{\delta}^{(n)}\right)$ and, for $\delta<\gamma, \mathscr{F}\left(T_{\delta}^{(n)}\right) \subset \mathscr{F}\left(T_{\gamma}^{(n)}\right), \mathscr{H}(n, \gamma)$ is a sub- $\sigma$-field of $\mathscr{F}\left(T_{\gamma}^{(n)}\right)$. Also, $\mathscr{H}(n, \gamma) \subset \mathscr{H}\left(n, r^{\prime}\right)$ whenever $\gamma<\gamma^{\prime}$.

LEMmA 3.1. If $m \leqq n$, then $\left\{T_{\alpha}^{(m)}=T_{\gamma}^{(n)}<\infty\right\} \in \mathscr{H}(n, \gamma)$.

Proof. We prove by induction on both $\gamma$ and $\alpha$. The induction hypothesis is that $\left\{T_{\gamma^{\prime}}^{(n)}=T_{\alpha^{\prime}}^{(m)}<\infty\right\} \in \mathscr{H}\left(n, \gamma^{\prime}\right)$ whenever $\gamma^{\prime}<\gamma$ and $\alpha^{\prime}<\pi$, or $\gamma^{\prime}=\gamma$ and $\alpha^{\prime}<\alpha$. Since the case $\alpha=0$ is trivial we assume $\alpha>0$. If $\alpha$ has a predecessor $\alpha^{\prime}$, then

$$
\begin{aligned}
\left\{T_{\gamma}^{(n)}=T_{\alpha}^{(m)}<\infty\right\}= & \bigcup_{\gamma^{\prime}<\gamma}\left(\{ T _ { \gamma ^ { \prime } } ^ { ( n ) } = T _ { \alpha ^ { \prime } } ^ { ( n ) } < \infty \} \cap \bigcup _ { i } \left\{X\left(T_{\gamma^{\prime}}^{(n)}\right) \in V_{i}^{(m)} ;\right.\right. \\
& \left.\left.X\left(T_{\delta}^{(n)}\right) \in U_{i}^{(m)} \text { for } \gamma^{\prime}<\delta<\gamma ; X\left(T_{\delta}^{(n)}\right) \in E-U_{i}^{(m)}, T_{\delta}^{(n)}<\infty\right\}\right) \\
& \in \mathscr{H}(n, \gamma) .
\end{aligned}
$$

If $\alpha$ has no predecessor, then $T_{\alpha}^{(m)}=\sup \left\{T_{\alpha^{\prime}}^{(m)} \mid \alpha^{\prime}<\alpha\right\}$. Now for $\alpha^{\prime}<\pi$,

$$
\left\{T_{\alpha^{\prime}}^{(m)}<T_{\gamma}^{(n)}<\infty\right\}=\left\{T_{\gamma}^{(n)}<\infty\right\} \cap \bigcup_{\gamma^{\prime}<\gamma}\left\{T_{\alpha^{\prime}}^{(m)}=T_{\gamma^{\prime}}^{(n)}<\infty\right\} \in \mathscr{H}(n, \gamma) .
$$

Hence if $\alpha^{\prime}<\pi$, then

$$
\left\{T_{\gamma}^{(n)}<T_{\alpha^{\prime}}^{(m)}\right\}=\left\{T_{\gamma}^{(n)}<\infty\right\}-\left\{T_{\alpha^{\prime}}^{(m)}=T_{\gamma}^{(n)}<\infty\right\}-\left\{T_{\alpha^{\prime}}^{(m)}<T_{\gamma}^{(n)}<\infty\right\} \in \mathscr{H}(n, \gamma) .
$$

It follows that

$$
\begin{aligned}
\left\{T_{\alpha}^{(m)}\right. & \left.=T_{\gamma}^{(n)}<\infty\right\}=\left\{T_{\gamma}^{(n)}<\infty\right\}-\left\{T_{\alpha}^{(m)}<T_{\gamma}^{(n)}<\infty\right\}-\left\{T_{\gamma}^{(n)}<T_{\alpha}^{(m)}\right\} \\
& =\left\{T_{\gamma}^{(n)}<\infty\right\}-\left\{T_{\alpha}^{(m)}<T_{\gamma}^{(n)}<\infty\right\}-\bigcup_{\alpha^{\prime}<\alpha}\left\{T_{\gamma}^{(n)}<T_{\alpha^{\prime}}^{(m)}\right\} \in \mathscr{H}(n, \gamma) .
\end{aligned}
$$

This completes the proof.

For the rest of this section let $m$ and $\alpha>0$ be fixed. For $n \geqq 0$ and $\gamma<\pi$ let $R_{\gamma}^{(n)}=\min \left\{T_{\gamma}^{(m+n)}, T_{\alpha}^{(m)}\right\}$. Note that for each $\mu$ there exists $\beta<\pi$ such that $P^{\mu}\left(R_{\beta}^{(n)}<T_{\alpha}^{(m)}\right)={ }^{*} P^{\mu}\left(R_{\beta}^{(n)}<T_{\alpha}^{(m)}\right)=0$. Let $\mathscr{H}_{\infty}(m, \alpha)$ be the $\sigma$-field generated by sets 
of the form $\left\{X\left(R_{\gamma}^{(n)}\right) \in B, R_{\gamma}^{(n)}<\infty\right\}$ or $\left\{X\left(T_{\alpha}^{(m)}\right) \in B, T_{\alpha}^{(m)}<\infty\right\}$, where $B \in \mathscr{B}_{\Delta}$ and $\gamma<\pi$. Let $\mathscr{H}_{\infty}(m, \alpha)$ be the smallest $\sigma$-field containing all $\mathscr{H}_{n}(m, \alpha), n \geqq 0$. Note that $\mathscr{H}_{\infty}(m, \alpha) \subset \mathscr{F}\left(T_{\alpha}^{(m)}\right)$.

CoRollaRY 3.2. $\mathscr{H}_{n}(m, \alpha) \cap\left\{T_{\alpha}^{(m)}=T_{\gamma}^{(m+n)}<\infty\right\} \subset \mathscr{H}(m+n, \gamma)$.

Proof. If $\Lambda=\left\{X\left(T_{\alpha}^{(m)}\right) \in B, T_{\alpha}^{(m)}<\infty\right\}$ then

$\Lambda \cap\left\{T_{\alpha}^{(m)}=T_{\gamma}^{(m+n)}<\infty\right\}=\left\{X\left(T_{\gamma}^{(m+n)}\right) \in B, T_{\alpha}^{(m)}=T_{\gamma}^{(m+n)}<\infty\right\} \in \mathscr{H}(m+n, \gamma)$

by Lemma 3.1. Let $\Lambda=\left\{X\left(R_{\gamma^{\prime}}^{(n)}\right) \in B, R_{\gamma^{\prime}}^{(n)}<\infty\right\}$. If $\gamma^{\prime} \leqq \gamma$ then

$\Lambda \cap\left\{T_{\alpha}^{(m)}=T_{\gamma}^{(m+n)}<\infty\right\}=\left\{X\left(T_{\gamma}^{(m+n)}\right) \in B, T_{\alpha}^{(m)}=T_{\gamma}^{(m+n)}<\infty\right\} \in \mathscr{H}(m+n, \gamma)$.

If $\gamma<\gamma^{\prime}$, then

$$
\Lambda \cap\left\{T_{\alpha}^{(m)}=T_{\gamma}^{(m+n)}<\infty\right\}=\left\{X\left(T_{\gamma^{\prime}}^{(m+n)}\right) \in B, T_{\alpha}^{(m)}=T_{\gamma}^{(m+n)}<\infty\right\} \in \mathscr{H}(m+n, \gamma) .
$$

The corollary follows.

LEMMA 3.3. $\mathscr{H}_{n}(m, \alpha)^{\mu} \subset \mathscr{H}_{n+1}(m, \alpha)^{\mu}$ for every $\mu$ and $n$.

Proof. (i) The following equalities show that for arbitrary $\delta$ the sets

$$
\left\{T_{\delta}^{(m+n+1)}<T_{\alpha}^{(m)}\right\} \text { and }\left\{T_{\delta}^{(m+n+1)}=T_{\alpha}^{(m)}<\infty\right\}
$$

are in $\mathscr{H}_{n+1}=\mathscr{H}_{n+1}(m, \alpha)$ :

$$
\begin{aligned}
&\left\{T_{\delta}^{(m+n+1)}<T_{\alpha}^{(m)}\right\}=\{\left.X\left(R_{\delta}^{(n+1}\right) \neq X\left(R_{\delta+1}^{(n+1)}\right), R_{\delta+1}^{(n+1)}<\infty\right\} \\
& \cup\left\{R_{\delta}^{(n+1)}<\infty, R_{\delta+1}^{(n+1)}=\infty\right\}, \\
&\left\{T_{\delta}^{(m+n+1)}=T_{\alpha}^{(m)}<\infty\right\}=\left\{X\left(R_{\delta^{\prime}}^{(n+1)}\right) \neq X\left(R_{\delta^{\prime}+1}^{(n+1)}\right) \text { for all } \delta^{\prime}<\delta,\right. \\
&\left.X\left(R_{\delta}^{(n+1)}\right)=X\left(R_{\delta+1}^{(n+1)}\right), R_{\delta+1}^{(n+1)}<\infty\right\} .
\end{aligned}
$$

(ii) Let us show that, if $\Lambda \in \mathscr{H}(m+n+1, \delta), \Lambda \cap\left\{T_{\delta}^{(m+n+1)}<T_{\alpha}^{(m)}\right\}$ and $\Lambda \cap\left\{T_{\delta}^{(m+n+1)}=T_{\alpha}^{(m)}<\infty\right\}$ are in $\mathscr{H}_{n+1}$. We need only to consider

$$
\Lambda=\left\{X\left(T_{\delta^{\prime}}^{(m+n+1)}\right) \in B, T_{\delta^{\prime}}^{(m+n+1)}<\infty\right\}
$$

where $B \in \mathscr{B}_{\Delta}, \delta^{\prime} \leqq \delta$. Now

$$
\begin{gathered}
\Lambda \cap\left\{T_{\delta}^{(m+n+1)}<T_{\alpha}^{(m)}\right\}=\left\{X\left(R_{\delta^{\prime}}^{(n+1)}\right) \in B, R_{\delta^{\prime}}^{(n+1)}<\infty\right\} \cap\left\{T_{\delta}^{(m+n+1)}<T_{\alpha}^{(n)}\right\}, \\
\Lambda \cap\left\{T_{\delta}^{(m+n+1)}=T_{\alpha}^{(m)}<\infty\right\}=\left\{X\left(R_{\delta^{\prime}}^{(n+1)}\right) \in B, R_{\delta^{\prime}}^{(n+1)}<\infty\right\} \\
\cap\left\{T_{\delta}^{(m+n+1)}=T_{\alpha}^{(m)}<\infty\right\},
\end{gathered}
$$

and it follows from (i) that they are $\mathscr{H}_{n+1}$-sets.

(iii) Suppose $\Lambda=\left\{X\left(R_{\gamma}^{(n)}\right) \in B, R_{\gamma}^{(n)}<\infty\right\}$ where $B \in \mathscr{B}_{\Delta}$ and $\gamma<\pi$. Then $\Lambda$ is the union of

and

$$
\Lambda_{1}=\left\{X\left(T_{\alpha}^{(m)}\right) \in B, T_{\alpha}^{(m)}<\infty\right\} \cap\left\{T_{\alpha}^{(m)} \leqq T_{\gamma}^{(m+n)}\right\}
$$

$$
\Lambda_{2}=\left\{X\left(T_{\gamma}^{(m+n)}\right) \in B, T_{\gamma}^{(m+n)}<\infty\right\} \cap\left\{T_{\gamma}^{(m+n)}<T_{\alpha}^{(m)}\right\}
$$


Let $\beta$ be such that $P^{\mu}\left(T_{\beta}^{(m+n+1)}<\infty\right)=0$. We have

$$
\begin{aligned}
& \Lambda_{1}=\Gamma_{1} \cup \bigcup_{\delta<\beta}\left(\left\{X\left(T_{\delta}^{(m+n+1)}\right) \in B, T_{\delta}^{(m+n+1)}<\infty\right\} \cap\left\{T_{\gamma}^{(m+n)} \geqq T_{\delta}^{(m+n+1)}<\infty\right\}\right. \\
& \left.\cap\left\{T_{\alpha}^{(m)}=T_{\delta}^{(m+n+1)}<\infty\right\}\right), \\
& \Lambda_{2}=\Gamma_{2} \cup \bigcup_{\delta<\beta}\left(\left\{X\left(T_{\delta}^{(m+n+1)}\right) \in B, T_{\delta}^{(m+n+1)}<\infty\right\} \cap\left\{T_{\gamma}^{(m+n)}=T_{\delta}^{(m+n+1)}<\infty\right\}\right. \\
& \left.\cap\left\{T_{\delta}^{(m+n+1)}<T_{\alpha}^{(m)}\right\}\right),
\end{aligned}
$$

where $\Gamma_{1}$ and $\Gamma_{2}$ are $\mathscr{F}$-sets contained in $\left\{T_{\beta}^{(m+n+1)}<\infty\right\}$. Now

$$
\left\{T_{\gamma}^{(m+n)}=T_{\delta}^{(m+n+1)}<\infty\right\}
$$

and

$$
\begin{aligned}
\left\{T_{\delta}^{(m+n+1)} \leqq T_{\gamma}^{(m+n)}, T_{\delta}^{(m+n+1)}<\infty\right\}= & \left\{T_{\gamma}^{(m+n)}=T_{\delta}^{(m+n+1)}<\infty\right\} \\
& \cup\left\{T_{\delta}^{(m+n+1)}<T_{\gamma}^{(m+n)}\right\}
\end{aligned}
$$

are in $\mathscr{H}(m+n+1, \delta)$ (see Lemma 3.1). It follows from (ii) that $\Lambda_{1}$ and $\Lambda_{2}$ are in $\mathscr{H}_{n+1}^{\mu}$ and hence so is $\Lambda$. This proves $\mathscr{H}_{n} \subset \mathscr{H}_{n+1}^{\mu}$ and the lemma follows.

LemMA 3.4. Let $K$ be a compact subset of $E$ and let $R_{m}=\inf \left\{T_{\alpha}^{(m)} \mid T_{\alpha}^{(m)} \geqq T_{K}\right\}$. If $x \notin K$, then $\left\{R_{m}=T_{\alpha}^{(m)}<\sigma\right\} \in \mathscr{H}_{\infty}(m, \alpha)^{\varepsilon_{x}} .\left(\varepsilon_{x}\right.$ is the unit mass at $x$.)

Proof. Since $\left\{R_{n}=T_{\alpha}^{(m)}<\sigma\right\}=\left\{T_{K} \leqq T_{\alpha}^{(m)}<\sigma\right\}-\bigcup_{\alpha^{\prime}<\alpha}\left\{T_{K} \leqq T_{\alpha^{\prime}}^{(m)}<\sigma\right\}$, it suffices to show that $\left\{T_{K} \leqq T_{\alpha^{\prime}}^{(m)}<\sigma\right\} \in \mathscr{H}_{\infty}(m, \alpha)^{\varepsilon}$ for all $\alpha^{\prime} \leqq \alpha$. Let $T_{n}=\inf \left\{T_{\gamma}^{(n)}<\sigma \mid\right.$ if $X\left(T_{y}^{(n)}\right) \in V_{i}^{(n)}$ then $\left.U_{i}^{(n)} \cap K \neq \varnothing\right\}$. By Proposition $2.5 T_{n} \uparrow T_{K}$ or $\sigma$ a.e. $P^{x}$. Hence we need only to show $\left\{T_{n} \leqq T_{\alpha^{\prime}}^{(m)}<\sigma\right\} \in \mathscr{H}_{\infty}(m, \alpha)^{\varepsilon}$ for $\alpha^{\prime} \leqq \alpha, n \geqq m$. Let $n \geqq m$ and let $\beta$ be such that $P^{x}\left(T_{\beta}^{(n)}<\infty\right)=0$. We have

$$
\left\{T_{n} \leqq T_{\alpha^{\prime}}^{(m)}<\sigma\right\}=\Gamma \cup \bigcup_{\gamma<\beta}\left\{T_{n}=T_{\gamma}^{(n)} \leqq T_{\alpha^{\prime}}^{(m)}<\sigma\right\}
$$

where $\Gamma$ is an $\mathscr{F}$-set contained in $\left\{T_{\beta}^{(n)}<\infty\right\}$. Now $\left\{T_{\gamma}^{(n)} \leqq T_{\alpha^{\prime}}^{(m)}<\sigma\right\}$ is the union of $\bigcup_{\gamma \leqq \delta<\beta}\left\{T_{\alpha^{\prime}}^{(m)}=T_{\delta}^{(n)}<\sigma\right\}$ and a subset (in $\mathscr{F}$ ) of $\left\{T_{\beta}^{(n)}<\infty\right\}$. Since for $\alpha^{\prime}<\alpha$

$$
\begin{aligned}
\left\{T_{\alpha^{\prime}}^{(m)}=T_{\delta}^{(n)}<\sigma\right\} & =\left\{T_{\alpha^{\prime}}^{(m)}=T_{\delta}^{(n)}<\sigma, T_{\delta}^{(n)}<T_{\alpha}^{(m)}\right\} \\
& \in \mathscr{H}(n, \delta) \cap\left\{T_{\delta}^{(n)}<T_{\alpha}^{(m)}\right\} \subset \mathscr{H}_{\infty}(m, \alpha)
\end{aligned}
$$

(see (ii) of the proof of Lemma 3.3), we have $\left\{T_{y}^{(n)} \leqq T_{\alpha^{\prime}}^{(m)}<\sigma\right\} \in \mathscr{H}_{\infty}(m, \alpha)^{\varepsilon} x$. Furthermore, $\left\{T_{n}=T_{\gamma}^{(n)} \leqq T_{\alpha}^{(m)}, T_{\gamma}^{(n)}<\sigma\right\}$ is clearly in $\mathscr{H}_{\infty}(m, \alpha)$ by the definition of $T_{n}$. It follows that $\left\{T_{n}=T_{y}^{(n)} \leqq T_{\alpha^{\prime}}^{(m)}<\sigma\right\} \in \mathscr{H}_{\infty}(m, \alpha)^{\varepsilon}$. (3.1) then implies $\left\{T_{n} \leqq T_{\alpha^{\prime}}^{(m)}<\sigma\right\}$ $\in \mathscr{H}_{\infty}(n, \alpha)^{\varepsilon}$. The proof is complete.

4. A martingale. In this section we make an additional hypothesis: (a) both $X$ and $X^{*}$ satisfy the stronger form of quasi-left continuity, i.e., if stopping times $T_{n}$ increase to $T$, then $X\left(T_{n}\right) \rightarrow X(T)$ a.e. (a.e.*) on $\{T<\infty\}$, and (b) $X(t) \rightarrow \Delta$ a.e. and a.e.* as $t \rightarrow \infty$. However, the following proposition depends only on hypothesis A.

Proposition 4.1. Given a partition $(\mathscr{U}, \mathscr{V})$ of $E$, there is a $\mathscr{B} \times \mathscr{B}$-measurable function $e: E \times E \rightarrow[0,1]$ such that $H_{E-U_{t}}^{*}(x, B)=\int_{B} e(x, y) H_{E-U_{t}}(x, d y)$ if $x \in V_{i}$. 
Proof. For $x \in E, B \in \mathscr{B}$ let $q(x, B)=H_{E-U_{t}}(x, B)$ and $q^{*}(x, B)=H_{E-U_{1}}^{*}(x, B)$, where $i$ is such that $x \in V_{i}$. For each $B, q(x, B)$ and $q^{*}(x, B)$ are $\mathscr{B}$-measurable in $x$. (This seems to be a standard fact; however, it is a special case of Proposition 5.1.) By hypothesis $\mathrm{A}, q^{*}(x, \cdot) \leqq q(x, \cdot)$ as measures on $\mathscr{B}$ and hence $q^{*}(x, \cdot)$ is absolutely continuous with respect to $q(x, \cdot)$. Since $\mathscr{B}$ is countably generated, it follows from a well-known theorem that there is a real-valued function $e$ defined on $E \times E$, measurable over $\mathscr{B} \times \mathscr{B}$, such that $q^{*}(x, B)=\int_{B} e(x, y) q(x, d y)$ for all $x \in E, B \in \mathscr{B}$. Obviously we may assume $0 \leqq e \leqq 1$.

Now for each $n$ let $e_{n}: E \times E \rightarrow[0,1]$ be a fixed $\mathscr{B} \times \mathscr{B}$-measurable function satisfying

$$
H_{E-U_{i}^{(n)}}^{*}(x, B)=\int_{B} e_{n}(x, y) H_{E-U_{!}^{(n)}}(x, d y)
$$

whenever $x \in V_{i}^{(n)}$ and $B \in \mathscr{B}$. Since $H_{E-U_{i}^{(n)}}\left(x, U_{i}^{(n)}\right)=0$ we may assume $e_{n}(x, x)=1$ for all $x \in E$. For $n \geqq 1$ and $0<\gamma<\pi$ let $M(n, \gamma)$ be a function on $\Omega$ defined as follows:

$$
\begin{aligned}
M(n, \gamma) & =0 & & \text { if } T_{\gamma}^{(n)} \geqq \sigma, \\
& =\prod_{\delta<\gamma} e_{n}\left(X\left(T_{\delta}^{(n)}\right), X\left(T_{\delta+1}^{(n)}\right)\right) & & \text { if } T_{\gamma}^{(n)}<\sigma,
\end{aligned}
$$

where the infinite product $\prod_{\delta<\gamma} e_{n}\left(X\left(T_{\delta}^{(n)}\right), X\left(T_{\delta+1}^{(n)}\right)\right)$ denotes the infimum of all finite products $e_{n}\left(X\left(T_{\delta_{1}}^{(n)}\right), X\left(T_{\delta_{1}+1}^{(n)}\right)\right) \cdots e_{n}\left(X\left(T_{\delta_{k}}^{(n)}\right), X\left(T_{\delta_{k}+1}^{(n)}\right)\right), \quad \delta_{1}<\cdots<\delta_{k}<\gamma$. Clearly $M(n, \gamma)$ is measurable over $\mathscr{H}(n, \gamma)$, and, if $\delta<\gamma, M(n, \gamma)=M(n, \delta)$ $\times\left[M\left(n ; \delta^{\prime}\right)\left(\theta_{\left.\left.T_{\delta}^{(n)}\right)\right]}\right.\right.$ on $\left\{T_{\delta}^{(n)}<\sigma\right\}$, where $\delta+\delta^{\prime}=\gamma$ (i.e., $\gamma$ is the $\delta^{\prime}$ th ordinal after $\delta$ ).

Proposition 4.2. For every $x \in E$ and for every $\Lambda \in \mathscr{H}(n, \gamma) \cap\left\{T_{y}^{(n)}<\sigma\right\}$

$$
{ }^{*} P^{x}(\Lambda)=E^{x}\{M(n, \gamma) ; \Lambda\} .
$$

Proof. (i) We first prove (4.1) for $\Lambda$ of the form $\left\{X\left(T_{0}^{(n)}\right) \in B_{0}, X\left(T_{y}^{(n)}\right) \in B_{1}, T_{y}^{(n)}<\sigma\right\}$. If $x \notin B_{0}$, both sides of (4.1) are zero. Hence we assume $x \in B_{0}$, in which case it suffices to let $\Lambda=\left\{X\left(T_{y}^{(n)}\right) \in B_{1}, T_{y}^{(n)}<\infty\right\}$. Let us define for $x \in E, 0<\delta<\pi$ measures $\nu_{x, \delta}$ and $\nu_{x, \delta}^{*}$ on $\mathscr{B}$ by setting $\nu_{x, \delta}(B)=E^{x}\left\{M(n, \delta) ; X\left(T_{\delta}^{(n)}\right) \in B, T_{\delta}^{(n)}<\sigma\right\}$ and $\nu_{x, \delta}^{*}(B)={ }^{*} P^{x}\left(X\left(T_{\delta}^{(n)}\right) \in B, T_{\delta}^{(n)}<\sigma\right)$. We show inductively $\nu_{x, \delta}=\nu_{x, \delta}^{*}$ for all $x$ and $\delta$. For $\delta=1$ we have, from the previous proposition,

$$
\begin{aligned}
& \nu_{x, 1}^{*}(B)=H_{E-U_{i}^{(n)}}^{*}(x, B)=\int_{B} e_{n}(x, y) H_{E-U_{i}^{(n)}}(x, d y) \\
& =E^{x}\left(e_{n}\left(X\left(T_{0}^{(n)}\right), X\left(T_{1}^{(n)}\right)\right) ; X\left(T_{1}^{(n)}\right) \in B, T_{1}^{(n)}<\sigma\right)=v_{x, 1}(B),
\end{aligned}
$$

$i$ being such that $x \in V_{i}^{(n)}$.

(ii) Suppose $\nu_{y, \delta^{\prime}}=\nu_{y, \delta^{\prime}}^{*}$ for all $y \in E$ and $0<\delta^{\prime}<\delta$. If $\delta$ has a predecessor $\delta^{\prime}>0$, then

$$
\begin{aligned}
& \nu_{x, \delta}^{*}(B)={ }^{*} E^{x}\left\{{ }^{*} P^{X\left(T_{o}^{(n)}\right)}\left(X\left(T_{1}^{(n)}\right) \in B, T_{1}^{(n)}<\sigma\right) ; T_{1}^{(n)}<\sigma\right\} \\
& =\int \nu_{y, 1}^{*}(B) \nu_{x, \delta^{\prime}}^{*}(d y)=\int v_{y, 1}(B) \nu_{x, \delta^{\prime}}(d y) \\
& =E^{x}\left\{M\left(n, \delta^{\prime}\right) E^{X\left(T_{\delta^{\prime}}^{(n)}\right)}\left[M(n, 1) ; X\left(T_{1}^{(n)}\right) \in B, T_{1}^{(n)}<\sigma\right] ; T_{\delta^{\prime}}^{(n)}<\sigma\right\} \\
& =E^{x}\left\{M\left(n, \delta^{\prime}\right)\left[M(n, 1)\left(\theta_{\left.T_{\delta}^{(n)}\right)}\right] ; X\left(T_{\delta}^{(n)}\right) \in B, T_{\delta}^{(n)}<\sigma\right\}=v_{x, \delta}(B)\right. \text {. }
\end{aligned}
$$


If $\delta$ has no predecessor, let $\delta_{1}<\cdots<\delta_{j} \uparrow \delta$. Then $T_{\delta_{j}}^{(n)} \uparrow T_{\delta}^{(n)}$ as $j \rightarrow \infty$. Hence $X\left(T_{\delta_{j}}^{(n)}\right) \rightarrow X\left(T_{\delta}^{(n)}\right)$ a.e. and a.e. ${ }^{*}$ on $\left\{T_{\delta}^{(n)}<\sigma\right\}$. Furthermore, the additional hypothesis implies that $X\left(T_{\delta_{j}}^{(n)}\right) \rightarrow \Delta$ a.e. and a.e.* on $\left\{T_{\delta_{j}}^{(n)}<\sigma\right.$ for all $\left.j, T_{\delta}^{(n)}=\sigma\right\}$ as $j \rightarrow \infty$. Hence for $f \in \mathscr{C}_{0}$ we have by the bounded convergence theorem, as $j \rightarrow \infty$,

$$
\begin{aligned}
\int f d \nu_{x, \delta_{j}}^{*}={ }^{*} E^{x}\left\{f\left(X\left(T_{\delta_{j}}^{(n)}\right)\right) ; T_{\delta_{j}}^{(n)}<\sigma\right\} & \rightarrow{ }^{*} E^{x}\left\{f\left(X\left(T_{\delta}^{(n)}\right)\right) ; T_{\delta}^{(n)}<\sigma\right\}=\int f d \nu_{x, \delta}^{*} \\
\int f d \nu_{x, \delta_{j}}=E^{x}\left\{f\left(X\left(T_{\delta_{j}}^{(n)}\right)\right) M\left(n, \delta_{j}\right) ;\right. & \left.T_{\delta_{j}}^{(n)}<\sigma\right\} \\
& \rightarrow E^{x}\left\{f\left(X\left(T_{\delta}^{(n)}\right)\right) M(n, \delta) ; T_{\delta}^{(n)}<\sigma\right\}=\int f d \nu_{x, \delta},
\end{aligned}
$$

since $M\left(n, \delta_{j}\right) \downarrow M(n, \delta)$ on $\left\{T_{\delta}^{(n)}<\sigma\right\}$. The condition $\nu_{x, \delta_{j}}^{*}=\nu_{x, \delta_{j}}$ for all $j$ then implies $\int f d \nu_{x, \delta}^{*}=\int f d \nu_{x, \delta}$ for all $f \in \mathscr{C}_{0}$. Hence $\nu_{x, \delta}^{*}=\nu_{x, \delta}$. This completes the proof that (4.1) holds for $\Lambda$ of the form $\left\{X\left(T_{\gamma}^{(n)}\right) \in B, T_{\gamma}^{(n)}<\sigma\right\}$.

(iii) We now prove (4.1) for $\Lambda=\left\{X\left(T_{\gamma_{j}}^{(n)}\right) \in B_{j}, j=1, \ldots, k ; X\left(T_{\gamma}^{(n)}\right) \in B, T_{\gamma}^{(n)}<\sigma\right\}$ where $\gamma_{1}<\cdots<\gamma_{k}<\gamma$ and $B, B_{j} \in \mathscr{B}$. We may assume $\gamma_{1}>0$ for otherwise (4.1) is trivial or reduces to this case. The proof is an induction on $k$. Note that the case $k=0$ is established already. Thus we assume $k>0$ and (4.1) holds when $\gamma$ is replaced by any $\delta>0$ and $\Lambda$ is replaced by any set of the form $\left\{X\left(T_{\delta_{j}}^{(n)}\right) \in A_{j}, j=1, \ldots, k-1\right.$; $\left.X\left(T_{\delta}^{(n)}\right) \in A, T_{\delta}^{(n)}<\sigma\right\}$, where $0<\delta_{1}<\cdots<\delta_{k-1}<\delta$ and $A, A_{j} \in \mathscr{B}$. Now with $\delta_{j}$, $j=1, \ldots, k-1$, and $\delta$ being such that $\gamma_{1}+\delta_{j}=\gamma_{1+j}$ and $\gamma_{1}+\delta=\gamma$, and with $\Lambda^{\prime}=\left\{X\left(T_{\delta_{j}}^{(n)}\right) \in B_{j+1}, j=1, \ldots, k-1 ; X\left(T_{\delta}^{(n)}\right) \in B, T_{\delta}^{(n)}<\sigma\right\}$, we have

$$
\begin{aligned}
* P^{x}(\Lambda) & ={ }^{*} E^{x}\left\{{ }^{*} P^{X\left(T_{\gamma_{1}}^{(n)}\right)}\left(\Lambda^{\prime}\right) ; X\left(T_{\gamma_{1}}^{(n)}\right) \in B_{1}, T_{\gamma_{1}}^{(n)}<\sigma\right\} \\
& =\int_{B_{1}} \nu_{x, \gamma_{1}}^{*}(d y)^{*} P^{y}\left(\Lambda^{\prime}\right)=\int_{B_{1}} \nu_{x, \gamma_{1}}(d y) E^{y}\left\{M(n, \delta) ; \Lambda^{\prime}\right\} \\
& =E^{x}\left\{M\left(n, \gamma_{1}\right) E^{X\left(T_{\gamma_{1}}^{(n)}\right)}\left[M(n, \delta) ; \Lambda^{\prime}\right] ; X\left(T_{\gamma_{1}}^{(n)}\right) \in B_{1}, T_{\gamma_{1}}^{(n)}<\sigma\right\} \\
& =E^{x}\left\{M ( n , \gamma _ { 1 } ) \left[M(n, \delta)\left(\theta_{T_{1}}^{(n)}\right) ; X\left(T_{\gamma_{j}}^{(n)}\right) \in B_{j}, j=1, \ldots, k,\right.\right. \\
& \left.\quad X\left(T_{\gamma}^{(n)}\right) \in B, T_{\gamma}^{(n)}<\sigma\right\} \\
& =E^{x}\{M(n, \gamma) ; \Lambda\} .
\end{aligned}
$$

Hence we have proved (4.1) for a big enough class of sets to guarantee that (4.1) holds for all $\Lambda \in \mathscr{H}(n, \gamma) \cap\left\{T_{\gamma}^{(n)}<\sigma\right\}$.

For each $m$ and $\alpha>0$ we define a sequence $\left\{M^{(n)}(m, \alpha), n=0,1,2, \ldots\right\}$ of functions on $\Omega$ by setting

$$
M^{(n)}(m, \alpha)=M(m+n, \lambda(m, \alpha, m+n)) .
$$

Recall that $\lambda(m, \alpha, m+n)=\inf \left\{\gamma \mid T_{\gamma}^{(m+n)}=T_{\alpha}^{(m)}\right\}$. Note that $M^{(n)}(m, \alpha)=0$ on $\left\{T_{\alpha}^{(m)} \geqq \sigma\right\}$.

LeMma 4.3. $M^{(n)}(m, \alpha)$ is measurable over $\mathscr{H}_{n}(m, \alpha)^{\mu}$ for every $\mu$.

Proof. $M^{(n)}(m, \alpha)=M(m+n, \gamma)$ on $\Lambda_{\gamma}=\left\{T_{\alpha}^{(m)}=T_{\gamma}^{(m+n)}<\infty\right\}$. Now

$$
\Lambda_{\gamma}=\left\{R_{\gamma+1}^{(n)}<\infty, X\left(R_{\gamma+1}^{(n)}\right)=X\left(R_{\gamma}^{(n)}\right) \neq X\left(R_{\delta}^{(n)}\right) \text { for all } \delta<\gamma\right\} \in \mathscr{H}_{n}(m, \alpha) .
$$


Hence $H(m+n, \gamma) \cap \Lambda_{y} \in \mathscr{H}_{n}(m, \alpha)$. Since $M(m+n, \gamma)$ is $\mathscr{H}(m+n, \gamma)$-measurable, its restriction to $\Lambda_{y}$ is measurable over $\mathscr{H}(m+n, \gamma) \cap \Lambda_{\gamma}$. It follows that the function $\sum_{y \leqq \beta} M^{(n)}(m, \alpha) I_{\Lambda_{y}}$ is $\mathscr{H}_{n}(m, \alpha)$-measurable for every $\beta$. $\left(I_{\Lambda}\right.$ is the indicator function of $\Lambda$.) But this function differs from $M^{(n)}(m, \alpha)$ on a subset of $\left\{R_{\beta}^{(n)}<T_{\alpha}^{(m)}\right\}$. Hence the lemma follows from the fact that there exists $\beta$ such that $\left\{R_{\beta}^{(n)}<T_{\alpha}^{(m)}\right\}$ is a $P^{\mu}$-null set.

COROLlaRY 4.4. $M^{(n)}(m, \alpha)$ is measurable over $\mathscr{F}\left(T_{\alpha}^{(m)}\right)$.

Proof. This follows from the previous lemma since $\mathscr{H}_{n}(m, \alpha) \subset \mathscr{F}\left(T_{\alpha}^{(m)}\right)$ and $\mathscr{F}\left(T_{\alpha}^{(m)}\right)=\bigcap_{\mu} \mathscr{F}\left(T_{\alpha}^{(m)}\right)^{\mu}$.

LeMma 4.5. For every $x \in E$ and $\Lambda \in \mathscr{H}_{n}(m, \alpha) \cap\left\{T_{\alpha}^{(m)}<\sigma\right\}$,

$$
{ }^{*} P^{x}(\Lambda)=E^{x}\left\{M^{(n)}(m, \alpha) ; \Lambda\right\} .
$$

Proof. Let $\beta<\pi$ be such that $P^{x}\left(R_{\beta}^{(n)}<T_{\alpha}^{(m)}\right)={ }^{*} P^{x}\left(R_{\beta}^{(n)}<T_{\alpha}^{(m)}\right)=0$. Then $\Lambda$ is the disjoint union of the sets $\Lambda \cap\left\{T_{\alpha}^{(m)}=T_{\gamma}^{(m+n)}<\infty\right\}, \gamma \leqq \beta$, and a set $\Gamma$ with $P^{x}(\Gamma)={ }^{*} P^{x}(\Gamma)=0$. Hence

and

$$
{ }^{*} P^{x}(\Lambda)=\sum_{\gamma \leqq \beta}{ }^{*} P^{x}\left(\Lambda \cap\left\{T_{\alpha}^{(m)}=T_{\gamma}^{(m+n)}<\infty\right\}\right)
$$

$$
E^{x}\left\{M^{(n)}(m, \alpha) ; \Lambda\right\}=\sum_{\gamma \leqq \beta} E^{x}\left\{M^{(n)}(m, \alpha) ; \Lambda \cap\left[T_{\alpha}^{(m)}=T_{\gamma}^{(m+n)}<\infty\right]\right\} .
$$

Now $\Lambda \cap\left\{T_{\alpha}^{(m)}=T_{\gamma}^{(m+n)}<\infty\right\} \in \mathscr{H}(m+n, \gamma)$ by Corollary 3.2, and on

$$
\Lambda \cap\left\{T_{\alpha}^{(m)}=T_{\gamma}^{(m+n)}<\infty\right\} \subset\left\{T_{\alpha}^{(m)}=T_{\gamma}^{(m+n)}<\sigma\right\},
$$

$M^{(n)}(m, \alpha)=M(m+n, \gamma)$ since $\lambda(m, \alpha, m+n)=\gamma$. Thus from Proposition 4.2 we have ${ }^{*} P^{x}\left(\Lambda \cap\left\{T_{\alpha}^{(m)}=T_{\gamma}^{(m+n)}<\infty\right\}\right)=E^{x}\left\{M^{(n)}(m, \alpha) ; \Lambda \cap\left[T_{\alpha}^{(m)}=T_{\gamma}^{(m+n)}<\infty\right]\right\}$. It follows that ${ }^{*} P^{x}(\Lambda)=E^{x}\left\{M^{(n)}(m, \alpha) ; \Lambda\right\}$.

We now prove the basic

THEOREM 4.6. For every $x$, the sequence of functions $M^{(n)}(m, \alpha), n=0,1,2, \ldots$, is a martingale with respect to the o-fields $\mathscr{H}_{n}(m, \alpha)^{\varepsilon_{x}}, n=0,1,2, \ldots$, and the measure $P^{x}$.

Proof. We have seen that $M^{(n)}=M^{(n)}(m, \alpha)$ is measurable over $\mathscr{H}_{n}(m, \alpha)^{\varepsilon_{x}}$ and $\mathscr{H}_{n}(m, \alpha)^{\varepsilon} \subset \mathscr{H}_{n+1}(m, \alpha)^{\varepsilon_{x}}$. Trivially $E^{x}\left\{M^{(n)}\right\}<\infty$ for all $n$. It remains to show that, for every $\Lambda \in \mathscr{H}_{n}(m, \alpha), E^{x}\left\{M^{(n)} ; \Lambda\right\}=E^{x}\left\{M^{(n+1)} ; \Lambda\right\}$. Since $M^{(n)}(m, \alpha)=0$ on $\left\{T_{\alpha}^{(m)} \geqq \sigma\right\}$, we may assume $\Lambda \subset\left\{T_{\alpha}^{(m)}<\sigma\right\}$. In view of Lemma 3.3 there exist $\Lambda_{1}$ and $\Lambda_{2}$ in $\mathscr{H}_{n+1}(m, \alpha)$ such that $\Lambda_{1} \subset \Lambda \subset \Lambda_{2} \subset\left\{T_{\alpha}^{(m)}<\sigma\right\}$ and $P^{x}\left(\Lambda_{1}\right)=P^{x}\left(\Lambda_{2}\right)$. By Lemma 4.5 we have

$$
\begin{aligned}
E^{x}\left\{M^{(n)} ; \Lambda\right\} & ={ }^{*} P^{x}(\Lambda) \leqq * P^{x}\left(\Lambda_{2}\right)=E^{x}\left\{M^{(n+1)} ; \Lambda_{2}\right\}=E^{x}\left\{M^{(n+1)} ; \Lambda\right\} \\
& =E^{x}\left\{M^{(n+1)} ; \Lambda_{1}\right\}={ }^{*} P^{x}\left(\Lambda_{1}\right) \leqq{ }^{*} P^{x}(\Lambda)=E^{x}\left\{M^{(n)} ; \Lambda\right\} .
\end{aligned}
$$

The theorem is proved.

For each pair $(m, \alpha), \alpha>0$, let $M^{\infty}(m, \alpha)$ (interchangeably $\left.M_{m, \alpha}^{\infty}\right)$ be defined by

$$
M^{\infty}(m, \alpha)=\liminf _{n \rightarrow \infty} M^{(n)}(m, \alpha) .
$$


Corollary 4.7. (i) For every $x, M^{(n)}(m, \alpha) \rightarrow M^{\infty}(m, \alpha)$ a.e. $P^{x}$ as $n \rightarrow \infty$; (ii) $0 \leqq M^{\infty}(m, \alpha) \leqq 1$ and $M^{\infty}(m, \alpha)=0$ on $\left\{T_{\alpha}^{(m)} \geqq \sigma\right\}$; (iii) $M^{\infty}(m, \alpha)$ is $\mathscr{F}\left(T_{\alpha}^{(m)}\right)$ measurable; (iv) for $\Lambda \in \mathscr{H}_{\infty}(m, \alpha)^{\varepsilon} \cap\left\{T_{\alpha}^{(m)}<\sigma\right\}, E^{x}\left\{M^{\infty}(m, \alpha) ; \Lambda\right\}={ }^{*} P^{x}(\Lambda)$.

Proof. (i) follows from Theorem 4.6 and the martingale convergence theorem, (ii) is trivial, and (iii) is a result of Corollary 4.4. If $\Lambda \in \mathscr{H}_{n}(m, \alpha)^{\varepsilon} \times\left\{T_{\alpha}^{(m)}<\sigma\right\}$, then $E^{x}\left\{M^{\left(n^{\prime}\right)}(m, \alpha) ; \Lambda\right\}={ }^{*} P^{x}(\Lambda)$ for all $n^{\prime} \geqq n$. As $n^{\prime} \rightarrow \infty$ we obtain from (i) that $E^{x}\left\{M^{\infty}(m, \alpha) ; \Lambda\right\}={ }^{*} P^{x}(\Lambda)$. (ii) follows from this and the fact that $\mathscr{H}_{n}(m, \alpha)^{\varepsilon}$ increases with $n$.

5. Removal of the additional hypothesis in 4. In this section we will obtain functions $M^{\infty}(m, \alpha)$ satisfying Corollary 4.7 without the additional hypothesis in the previous section.

LEMMA 5.1. Let $\phi$ be a bounded real-valued $\mathscr{H}(n, \gamma)$-measurable function. Then $E^{x}\left\{\phi ; T_{\gamma}^{(n)}<\sigma\right\}$ and ${ }^{*} E^{x}\left\{\phi ; T_{\gamma}^{(n)}<\sigma\right\}$ are $\mathscr{B}_{\Delta}$-measurable in $x$.

Proof. Let $W(m, i)=\left\{y \in E \mid \rho\left(y, E-U_{i}^{(n)}\right)<1 / m\right\}$. Let $\bar{Q}_{m}=T_{W(m, i)}$ if $X(0) \in V_{i}^{(n)}$ and $=\infty$ if $X(0)=\Delta . \bar{Q}_{m}$ is $\mathscr{G}$-measurable; hence so is the increasing limit $Q_{1}$ of $\bar{Q}_{m}$ as $m \rightarrow \infty$. Since $\left\{X\left(Q_{1}\right) \in B, Q_{1}<\infty\right\} \in \mathscr{G}$ for $B \in B_{\Delta}$, it follows from the way $Q_{1}$ is defined that $Q_{2}$ defined by $Q_{2}=Q_{1}+Q_{1}\left(\theta_{Q_{1}}\right)$ for $Q_{1}<\infty$ and $=\infty$ for $Q_{1}=\infty$ is $\mathscr{G}$-measurable. In fact, let $\bar{Q}_{m}^{\prime}=\inf \left\{t \geqq Q_{1} \mid X(t) \in W(m, i)\right\}$ if $X\left(Q_{1}\right) \in V_{i}^{(n)}$, and $=\infty$ if otherwise; then $\bar{Q}_{m}^{\prime}$ are $\mathscr{G}$-measurable and increase to $Q_{2}$. From the same reasoning we see that all the $Q_{\gamma}, \gamma \leqq \pi$, defined below are $\mathscr{G}$-measurable:

$$
\begin{aligned}
Q_{\gamma+1} & =Q_{\gamma}+Q_{1}\left(\theta_{Q_{\gamma}}\right) & & \text { if } Q_{\alpha}<\infty, \\
& =\infty & & \text { if otherwise, } \\
Q_{\gamma} & =\sup _{\delta<\gamma} Q_{\delta} & & \text { if } \gamma \text { is a limit ordinal. }
\end{aligned}
$$

Now it follows from the quasi-left continuity that $\left\{T_{1}^{(n)}<\sigma\right\} \subset\left\{Q_{1}<\sigma\right\}$ and $T_{1}^{(n)}=Q_{1}$ a.e. and a.e. ${ }^{*}$ on $\left\{Q_{1}<\sigma\right\}$. By the definitions of $T_{\alpha}^{(n)}$ and $Q_{\alpha}$ we then have $T_{\alpha}^{(n)}=Q_{\alpha}$ a.e. and a.e.* on $\left\{T_{\alpha}^{(n)}<\sigma\right\} \cup\left\{Q_{\alpha}<\sigma\right\}$. The lemma immediately follows.

Let $n$ be fixed for a while. Denote by $\gamma$ the set of limit ordinals $\leqq \gamma$.

We will define inductively functions $\xi_{\gamma}^{(n)}: \Omega \rightarrow[0,1], \gamma$ a limit ordinal, satisfying:

(i) $\xi_{\gamma}^{(n)}$ is measurable over $\mathscr{H}(n, \gamma)$,

(ii) if $\delta+\gamma^{\prime}=\gamma$ then $\xi_{\gamma^{\prime}}^{(n)}\left(\theta_{T_{\delta}^{(n)}}\right)=\xi_{\gamma}^{(n)}$ a.e. on $\left\{T_{\delta}^{(n)}<\sigma\right\}$,

(iii) for every $x \in E$ and $\Lambda \in \mathscr{H}(n, \gamma) \cap\left\{T_{\gamma}^{(n)}<\sigma\right\},{ }^{*} P^{x}(\Lambda)$

$$
=E^{x}\{M(n, \gamma) ; \Lambda\}
$$

where $M(n, \gamma)$ is defined as follows (for every $\gamma<\pi)$ :

$$
\begin{aligned}
M(n, \gamma) & =\left[\prod_{\delta<\gamma} e_{n}\left(X\left(T_{\delta}^{(n)}\right), X\left(T_{\delta+1}^{(n)}\right)\right)\right]\left[\prod_{\delta \in \gamma} \xi_{\delta}^{(n)}\right], & & T_{\gamma}^{(n)}<\sigma, \\
& =0, & & T_{\gamma}^{(n)} \geqq \sigma,
\end{aligned}
$$

$\prod_{\delta \in \gamma} \xi_{\delta}^{(n)}$ meaning 1 if $\gamma$ is finite. If $\gamma^{\prime}=\gamma+p, p$ a finite ordinal, then we can obtain from (iii) of (5.1) that

$$
{ }^{*} P^{x}(\Lambda)=E^{x}\left\{M\left(n, \gamma^{\prime}\right) ; \Lambda\right\} \text { for every } x \in E \text { and } \Lambda \in \mathscr{H}\left(n, \gamma^{\prime}\right) \cap\left\{T_{\gamma^{\prime}}^{(n)}<\sigma\right\}
$$


This is obvious from the proof of Proposition 4.2. Hence in order to define $\xi_{y}^{(n)}, \gamma$ a limit ordinal, we may suppose that $\xi_{\delta}^{(n)}$ are defined and satisfy (5.1) for $\delta \in \gamma-\{\gamma\}$ and (5.2) holds for all $\gamma^{\prime}<\gamma$. Let $\gamma_{1}<\cdots<\gamma_{m} \uparrow \gamma$. Let $M^{\prime}(n, \gamma)=\lim _{m} M\left(n, \gamma_{m}\right)$.

LemMA 5.2. For each $x,{ }^{*} P^{x}\left(X\left(T_{\gamma}^{(n)}\right) \in d y, T_{\gamma}^{(n)}<\sigma\right)=E^{x}\left\{M^{\prime}(n, \gamma) ; X\left(T_{\gamma}^{(n)}\right) \in d y\right.$, $\left.T_{\gamma}^{(n)}<\sigma\right\}$ as measures on $\mathscr{B}$.

Proof. Suppose as $m \rightarrow \infty X\left(T_{\gamma_{1}}^{(n)}\right) \rightarrow X\left(T_{\gamma}^{(n)}\right) \in V_{i}^{(n)}$. Then there exists $m$ ( $m$ depening on $\omega$ ) such that $X\left(T_{\gamma^{\prime}}^{(n)}\right) \in U_{i}^{(n)}$ for $\gamma_{m} \leqq \gamma^{\prime}<\gamma$. Necessarily $X\left(T_{\gamma^{\prime}}^{(n)}\right) \in V_{j}^{(n)} \cap U_{i}^{(n)}$ for some $j \neq i$ if $\gamma_{m} \leqq \gamma^{\prime}<\gamma$. Now if $V_{j}^{(n)} \cap U_{i}^{(n)} \neq \varnothing$ and $j \neq i$, then $U_{j}^{(n)} \cap V_{i}^{(n)}=\varnothing$ by conditions (ii) and (iii) of Proposition 2.1. This means that for all large $m$, $T_{\gamma}^{(n)}=T_{V_{i}^{(n)}}\left(\theta_{T_{\gamma_{m}}^{(n)}}\right)+T_{\gamma_{m}}^{(n)}$ on $\left\{X\left(T_{\gamma}^{(n)}\right) \in V_{i}^{(n)}, T_{\gamma}^{(n)}<\sigma\right\}$. Let

$$
O_{k}=\left\{y \in U_{i}^{(n)} \mid 0<\rho\left(y, V_{i}^{(n)}\right) \leqq 1 / k\right\} .
$$

Now for a Borel $B \subset V_{i}^{(n)}$ we have from the induction hypothesis

$$
\begin{aligned}
& { }^{*} E^{x}\left\{H_{\left(E-U_{l}^{(n)}\right) \cup V_{l}^{(n)}}\left(X\left(T_{\gamma_{m}^{\prime}}^{(n)}\right), B\right) ; X\left(T_{\gamma^{\prime}}^{(n)}\right) \in O_{k} \text { for } \gamma_{m} \leqq \gamma^{\prime} \leqq \gamma_{m^{\prime}}, T_{\gamma_{m^{\prime}}}^{(n)}<\sigma\right\} \\
& \quad \leqq E^{x}\left\{M\left(n, \gamma_{m^{\prime}}\right) H_{\left(E-U_{l}^{(n)}\right) \cup V_{l}^{(n)}}\left(X\left(T_{\gamma_{m}^{\prime}}^{(n)}\right), B\right) ; X\left(T_{\gamma^{\prime}}^{(n)}\right) \in O_{k} \text { for } \gamma_{m} \leqq \gamma^{\prime} \leqq \gamma_{m^{\prime}}, T_{\gamma_{m^{\prime}}}^{(n)}<\sigma\right\} .
\end{aligned}
$$

Let $m^{\prime} \rightarrow \infty$ to obtain by the bounded convergence theorem

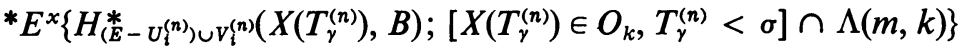

$$
\begin{aligned}
& +{ }^{*} P^{x}\left(\left\{X\left(T_{\gamma}^{(n)}\right) \in B, T_{\gamma}^{(n)} \in B, T_{\gamma}^{(n)}<\sigma\right\} \cap \Lambda(m, k)\right)
\end{aligned}
$$

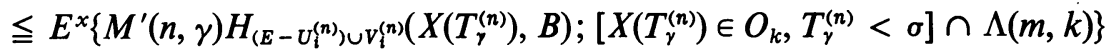

$$
\begin{aligned}
& +E^{x}\left\{M^{\prime}(n, \gamma) ;\left[X\left(T_{\gamma}^{(n)}\right) \in B, T_{\gamma}^{(n)}<\sigma\right] \cap \Lambda(m, k)\right\}
\end{aligned}
$$

where $\Lambda(m, k)=\left\{X\left(T_{\gamma^{\prime}}^{(n)}\right) \in O_{k}\right.$ for $\left.\gamma_{m} \leqq \gamma^{\prime}<\gamma, T_{\gamma}^{(n)}<\sigma\right\}$. As $m \rightarrow \infty$, we obtain in the limit

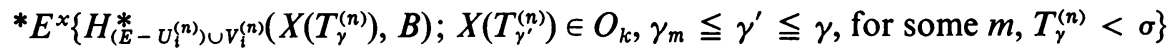

$$
\begin{aligned}
& +{ }^{*} P^{x}\left(X\left(T_{\gamma}^{(n)}\right) \in B, T_{\gamma}^{(n)}<\sigma\right)
\end{aligned}
$$

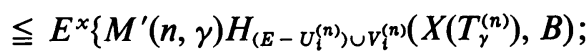

$$
\begin{aligned}
& \left.X\left(T_{\gamma^{\prime}}^{(n)}\right) \in O_{k}, \gamma_{m} \leqq \gamma^{\prime} \leqq \gamma, \text { for some } m, T_{\gamma}^{(n)}<\sigma\right\} \\
& +E^{x}\left\{M^{\prime}(n, \gamma) ; X\left(T_{\gamma}^{(n)}\right) \in B, T_{\gamma}^{(n)}<\sigma\right\} .
\end{aligned}
$$

Let $k \rightarrow \infty$. Then the first terms on both sides of the above inequality approach 0 . Thus we have ${ }^{*} P^{x}\left(X\left(T_{\gamma}^{(n)}\right) \in B, T_{\gamma}^{(n)}<\sigma\right) \leqq E^{x}\left\{M^{\prime}(n, \gamma) ; X\left(T_{\gamma}^{(n)}\right) \in B, T_{\gamma}^{(n)}<\sigma\right\}$ for $B \subset V_{i}^{(n)}$. The lemma follows.

LEMmA 5.3. There exists a function $g_{\gamma}: E \times E \rightarrow[0,1]$, measurable over $\mathscr{B} \times \mathscr{B}$, such that

$$
{ }^{*} P^{x}\left(X\left(T_{\gamma}^{(n)}\right) \in B, T_{\gamma}^{(n)}<\sigma\right)=E^{x}\left\{g_{\gamma}\left(X(0), X\left(T_{\gamma}^{(n)}\right)\right) M^{\prime}(n, \gamma) ; X\left(T_{\gamma}^{(n)}\right) \in B, T_{\gamma}^{(n)}<\sigma\right\}
$$

for all $x \in E, B \in \mathscr{B}$. 
Proof. It follows from Lemma 5.1 that

$$
{ }^{*} P^{x}\left(X\left(T_{\gamma}^{(n)}\right) \in B, T_{\gamma}^{(n)}<\sigma\right)
$$

and

$$
E^{x}\left\{M^{\prime}(n, \gamma) ; X\left(T_{\gamma}^{(n)}\right) \in B, T_{\gamma}^{(n)}<\sigma\right\}
$$

are $\mathscr{B}$-measurable in $x$ for each $B \in \mathscr{B}$.

Lemma 5.2 then implies the existence of a function $g_{\gamma}: E \times E \rightarrow[0,1]$, measurable over $\mathscr{B} \times \mathscr{B}$, such that

$$
\begin{aligned}
{ }^{*} P^{x}\left(X\left(T_{\gamma}^{(n)}\right) \in B, T_{\gamma}^{(n)}<\sigma\right) & =\int_{B} g_{\gamma}(x, y) E^{x}\left\{M^{\prime}(n, \gamma) ; X\left(T_{\gamma}^{(n)}\right) \in d y, T_{\gamma}^{(n)}<\sigma\right\} \\
& =E^{x}\left\{g_{\gamma}\left(X(0), X\left(T_{\gamma}^{(n)}\right) M^{\prime}(n, \gamma) ; X\left(T_{\gamma}^{(n)}\right) \in B, T_{\gamma}^{(n)}<\sigma\right\}\right. \\
& \text { for all } x \in E \text { and } B \in \mathscr{B} .
\end{aligned}
$$

Let $\delta_{m}$ be such that $\gamma_{m}+\delta_{m}=\gamma$. Suppose $g_{\delta_{m}}$ is also defined and satisfies Lemma 5.3 with $\delta_{m}$ replacing $\gamma$ throughout. Let $\eta_{m}=g_{\delta_{m}}\left(X\left(T_{\gamma_{m}}^{(n)}\right), X\left(T_{\gamma}^{(n)}\right)\right)$ on $\left\{T_{\gamma}^{(n)}<\sigma\right\}$ and $=0$ elsewhere. Let $\mathscr{H}^{\prime}\left(n, \gamma_{m}\right)$ be the $\sigma$-field generated by $\mathscr{H}\left(n, \gamma_{m}\right)$ and sets of the form $\left\{X\left(T_{\gamma}^{(n)}\right) \in B, T_{\gamma}^{(n)}<\sigma\right\}$.

Proposition 5.4. For every $x,\left\{\eta_{m}, m=1,2, \ldots\right\}$ is a martingale with respect to the measure $M^{\prime}(n, \gamma) d P^{x}$ and the $\sigma$-fields $\mathscr{H}^{\prime}\left(n, \gamma_{m}\right)$.

Proof. It suffices to show that for $\Lambda=\Lambda_{1} \cap\left\{X\left(T_{y}^{(n)}\right) \in B, T_{\gamma}^{(n)}<\sigma\right\}$, where $\Lambda_{1} \in \mathscr{H}\left(n, \gamma_{m}\right) \cap\left\{T_{\gamma_{m}}^{(n)}<\sigma\right\}$ and $B \in \mathscr{B}, E^{x}\left\{\eta_{m} M^{\prime}(n, \gamma) ; \Lambda\right\}={ }^{*} P^{x}(\Lambda)$. From the previous proposition and the induction hypotheses we have

$$
\begin{aligned}
E^{x}\left\{\eta_{m} M^{\prime}(n, \gamma) ; \Lambda\right\}= & E^{x}\left\{M ( n , \gamma _ { m } ) E ^ { X ( T _ { \gamma m } ^ { ( n ) } ) } \left[g_{\delta_{m}}\left(X(0), X\left(T_{\gamma}^{(n)}\right)\right) M^{\prime}(n, \gamma) ;\right.\right. \\
& \left.\left.X\left(T_{\gamma}^{(n)}\right) \in B, T_{\gamma}^{(n)}<\sigma\right] ; \Lambda_{1}\right\} \\
& ={ }^{*} E^{x}\left\{{ }^{*} P^{X\left(T_{\gamma m}^{(n)}\right)}\left(X\left(T_{\gamma}^{(n)}\right) \in B, T_{\gamma}^{(n)}<\sigma\right) ; \Lambda_{1}\right\} \\
& ={ }^{*} P^{x}\left(\Lambda_{1} \cap\left\{X\left(T_{\gamma}^{(n)}\right) \in B, T_{\gamma}^{(n)}<\sigma\right\}\right)={ }^{*} P^{x}(\Lambda) .
\end{aligned}
$$

Let $\xi_{y}^{(n)}=\lim \inf _{m \rightarrow \infty} \eta_{m}$. Then by the martingale convergence theorem $\eta_{m} \rightarrow \xi_{y}^{(n)}$ a.e. as $m \rightarrow \infty$. We claim that $\xi_{\gamma}^{(n)}$ satisfies (5.1). (i) of (5.1) is obvious, and (iii) is a result of the facts that $\eta_{m} \rightarrow \xi_{\gamma}^{(n)}$ a.e. and that $E^{x}\left\{\eta_{m} M^{\prime}(n, \gamma) ; \Lambda\right\}={ }^{*} P^{x}(\Lambda)$ for $\Lambda \in \mathscr{H}^{\prime}\left(n, \gamma_{p}\right) \cap\left\{T_{\gamma}^{(n)}<\sigma\right\}, p \leqq m$. To show (ii), note that

$$
\xi_{\gamma^{\prime}}^{(n)}\left(\theta_{\left.T_{\delta}^{(n)}\right)}=\liminf _{m \rightarrow \infty} g_{\delta_{m}^{\prime}}\left(X\left(T_{\delta+\gamma_{m}^{\prime}}^{(n)}\right), X\left(T_{\gamma}^{(n)}\right)\right)\right.
$$

on $\left\{T_{\gamma}^{(n)}<\sigma\right\}$, where $\gamma_{1}^{\prime}<\cdots<\gamma_{m}^{\prime} \uparrow \gamma^{\prime}$ and $\gamma_{m}^{\prime}+\delta_{m}^{\prime}=\gamma^{\prime}$. Let $\left\{\gamma_{m}^{\prime \prime}\right\}$ be the increasing sequence formed by $\left\{\gamma_{m}\right\}$ and $\left\{\delta+\gamma_{m}^{\prime}\right\}$ and let $\gamma_{m}^{\prime \prime}+\delta_{m}^{\prime \prime}=\gamma$. Then Proposition 5.4 implies $g_{\delta_{m}^{\prime \prime}}\left(X\left(T_{\gamma_{m}^{\prime \prime}}^{(n)}\right), X\left(T_{\gamma}^{(n)}\right)\right)$ converges a.e. on $\left\{T_{\gamma}^{(n)}<\sigma\right\}$. This proves $\xi_{\gamma^{\prime}}^{(n)}\left(\theta_{T_{\delta}^{(n)}}\right)=\xi_{\gamma}^{(n)}$ a.e. on $\left\{T_{y}^{(n)}<\infty\right\}$.

Thus by induction we have the following proposition, in which $\gamma$ need not be a limit ordinal because of a previous remark. 
Proposition 5.5. $M(n, \gamma)$ is $\mathscr{H}(n, \gamma)$-measurable; for each $x$ and $\Lambda \in \mathscr{H}(n, \gamma)$ $\cap\left\{T_{\gamma}^{(n)}<\sigma\right\}, E^{x}\{M(n, \gamma) ; \Lambda\}={ }^{*} P^{x}(\Lambda)$. Let $M^{(n)}(m, \alpha)=M(m+n, \lambda(m, \alpha, m+n))$ for $n \geqq 0$ and

$$
M^{\infty}(m, \alpha)=\liminf _{n \rightarrow \infty} M^{(n)}(m, \alpha) .
$$

Then Lemma 4.3, Corollary 4.4, Lemma 4.5, Theorem 4.6 and finally Corollary 4.7 again hold, since their proofs depend only on the properties of $M(n, \gamma)$ stated in the above proposition.

6. Properties of the functions $M^{\infty}(m, \alpha)$.

LeMMA 6.1. For any two pairs $(m, \alpha)$ and $\left(m^{\prime}, \alpha^{\prime}\right), M^{\infty}(m, \alpha)=M^{\infty}\left(m^{\prime}, \alpha^{\prime}\right)$ on $\left\{T_{\alpha}^{(m)}=T_{\alpha^{\prime}}^{\left(m^{\prime}\right)}\right\}$.

Proof. Suppose $m \leqq m^{\prime}$. If $T_{\alpha}^{(m)}=T_{\alpha^{\prime}}^{\left(m^{\prime}\right)}$, then

$$
\begin{aligned}
\lambda\left(m^{\prime}, \alpha^{\prime}, m^{\prime}+n\right) & =\inf \left\{\gamma \mid T_{\gamma}^{\left(m^{\prime}+n\right)}=T_{\alpha^{\prime}}^{\left(m^{\prime}\right)}\right\} \\
& =\inf \left\{\gamma \mid T_{\gamma}^{\left(m+m^{\prime}-m+n\right)}=T_{\alpha}^{(m)}\right\}=\lambda\left(m, \alpha, m+\left(m^{\prime}-m+n\right)\right) .
\end{aligned}
$$

Hence

$$
\begin{aligned}
M^{\left(m^{\prime}-m+n\right)}(m, \alpha) & =M\left(m+\left(m^{\prime}-m+n\right), \lambda\left(m, \alpha, m+\left(m^{\prime}-m+n\right)\right)\right) \\
& =M\left(m^{\prime}+n, \lambda\left(m^{\prime}, \alpha^{\prime}, m^{\prime}+n\right)\right) \\
& =M^{(n)}\left(m^{\prime}, \alpha^{\prime}\right)
\end{aligned}
$$

for all $n$ on $\left\{T_{\alpha}^{(m)}=T_{\alpha^{\prime}}^{\left(m^{\prime}\right)}<\sigma\right\}$, while on $\left\{T_{\alpha}^{(m)}=T_{\alpha^{\prime}}^{\left(m^{\prime}\right)} \geqq \sigma\right\}$,

$$
M^{(n)}(m, \alpha)=M^{(n)}\left(m^{\prime}, \alpha^{\prime}\right)=0 .
$$

The lemma then follows from the definitions of $M^{\infty}(m, \alpha)$ and $M^{\infty}\left(m^{\prime}, \alpha^{\prime}\right)$.

For $\omega \in \Omega$, let $J(\omega)=\left\{t \in[0, \infty] \mid t=T_{\alpha}^{(m)}(\omega)\right.$ for some $\left.(m, \alpha)\right\}$. Let $M^{\infty}(m, 0) \equiv 1$ on $\Omega$ for all $m$. For $\omega \in \Omega$ let $M(\cdot, \omega)$ be defined on $J(\omega)$ by

$$
M(t, \omega)=M_{m, \alpha}^{\infty}(\omega),
$$

where $(m, \alpha)$ is any pair satisfying $T_{\alpha}^{(m)}(\omega)=t . M(\cdot, \omega)$ is well defined because of Lemma 6.1. For any random variable $T$ with $T(\omega) \in J(\omega)$ for all $\omega$, the function $M(T)$, interchangeably $M_{T}$, is defined by $M(T)(\omega)=M(T(\omega), \omega)$. In particular we have $M\left(T_{\alpha}^{(m)}\right)=M^{\infty}(m, \alpha)$. We will investigate some properties of $M(\cdot, \omega)$ before setting up the desired multiplicative functional.

LemMa 6.2. For each $\omega, M(\cdot, \omega)$ is nonincreasing on $J(\omega)$.

Proof. Let $0<\alpha<\alpha^{\prime}$. It follows from definition that $M^{(n)}(m, \alpha) \geqq M^{(n)}\left(m, \alpha^{\prime}\right)$. Hence $M^{\infty}(m, \alpha) \geqq M^{\infty}\left(m, \alpha^{\prime}\right)$. Since $M^{\infty}(m, 0) \equiv 1$ and $M^{\infty}(m, \alpha) \leqq 1$, we have that, for each $\omega, M(\cdot, \omega)$ is nonincreasing on $J_{m}(\omega)=\left\{t \in[0, \infty] \mid t=T_{\alpha}^{(m)}(\omega)\right.$ for some $\alpha$. The fact that $J_{m}(\omega) \uparrow J(\omega)$ implies the lemma.

Lemma 6.3. Let $x \in I$. Then $M\left(T_{1}^{(m)}\right) \rightarrow 1$ a.e. $P^{x}$ as $m \rightarrow \infty$. 
Proof. Since $I=I^{*}$ (Proposition 1.1), $x$ is also in $I^{*}$. Let $i(m)$ be such that $x \in V_{i(m)}^{(m)}$. Then, since $U_{i(m)}^{(m)} \downarrow\{x\},{ }^{*} P^{x}\left(T_{1}^{(m)}<\infty\right)={ }^{*} P^{x}\left(T_{E-U_{i(m)}^{(m)}}^{(m)}<\right) \uparrow 1$ as $m \rightarrow \infty$. Now by Corollary $4.7 E^{x}\left\{M\left(T_{1}^{(m)}\right) ; T_{1}^{(m)}<\infty\right\}={ }^{*} P^{x}\left(T_{1}^{(m)}<\infty\right)$. It then follows from the previous lemma that $M\left(T_{1}^{(m)}\right) \rightarrow 1$ a.e. $P^{x}$.

LEMMA 6.4. $M\left(T_{\alpha+\alpha^{\prime}}^{(m)}\right)=M\left(T_{\alpha}^{(m)}\right)\left[M\left(T_{\alpha^{\prime}}^{(m)}\right)\left(\theta_{\left.T_{\alpha}^{(m)}\right)}\right]\right.$ a.e. on $\left\{T_{\alpha}^{(m)}<\infty\right\}$ for arbitrary $m, \alpha$ and $\alpha^{\prime}$.

Proof. Obviously we may assume $\alpha, \alpha^{\prime}>0$. On $\left\{T_{\alpha}^{(m)}<\infty\right\}, M^{(n)}\left(m, \alpha+\alpha^{\prime}\right)$

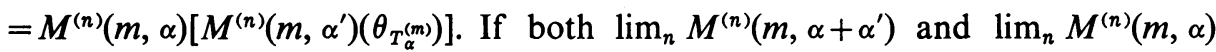
exist, in which case they are equal to $M\left(T_{\alpha+\alpha^{\prime}}^{(m)}\right)$ and $M\left(T_{\alpha}^{(m)}\right)$ respectively, then so does $\lim _{n}\left[M^{(n)}\left(m, \alpha^{\prime}\right)\left(\theta_{\left.\left.T_{\alpha}^{(m)}\right)\right]}\right.\right.$ and it is equal to $M\left(T_{\alpha^{\prime}}^{(m)}\right)\left(\theta_{\left.T_{\alpha}^{(m)}\right)}\right.$. Hence the lemma follows from (i) of Corollary 4.7.

COROllaRY 6.5. For arbitrary pairs $(m, \alpha)$ and $\left(m^{\prime}, \alpha^{\prime}\right)$,

$$
M\left(T_{\alpha}^{(m)}+T_{\alpha^{\prime}}^{\left(m^{\prime}\right)}\left(\theta_{\left.\left.T_{\alpha}^{(m)}\right)\right)}=M\left(T_{\alpha}^{(m)}\right)\left[M\left(T_{\alpha^{\prime}}^{\left(m^{\prime}\right)}\right)\left(\theta_{\left.T_{\alpha}^{(m)}\right)}\right]\right.\right.\right.
$$

a.e. on $\left\{T_{\alpha}^{(m)}<\infty\right\}$.

Proof. This follows from Lemma 6.5 and the facts that, with $m^{\prime \prime}=\max \left\{m, m^{\prime}\right\}$,

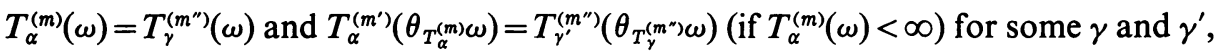
and that for a fixed $x$, there is a $\beta$ such that $P^{x}\left\{T_{\beta}^{\left(m^{\prime \prime}\right)}<\infty\right\}=0$.

Proposition 6.6. $M(\cdot, \omega)$ is right continuous on $J(\omega)$ a.e. $P^{x}(d \omega)$ for every $x$.

Proof. We show first that for every $x, M(\cdot, \omega)$ is right continuous at 0 a.e. $P^{x}(d \omega)$. If $x \in I$, this follows from Lemmas 6.3 and 6.4. If $x \in H$, it follows from the fact that, a.e. $P^{x}(d \omega)$, there exists $\delta>0$ such that $T_{1}^{(m)}(\omega)>\delta$ for all $m$. Now let $(m, \alpha)$ be fixed. The strong Markov property and the above fact imply that $M\left(T_{1}^{\left(m^{\prime}\right)}\right)\left(\theta_{T_{\alpha}^{(m)}}\right) \rightarrow 1$ a.e. on $\left\{T_{\alpha}^{(m)}<\infty, T_{1}^{\left(m^{\prime}\right)}\left(\theta_{\left.T_{\alpha}^{(m)}\right)} \downarrow 0\right.\right.$ as $\left.m^{\prime} \rightarrow \infty\right\}$. It then follows from Corollary 6.5 that $M(\cdot, \omega)$ is right continuous at $T_{\alpha}^{(m)}(\omega)$ a.e. $P^{x}(d \omega)$ for every $x$. Since for a fixed $x$ there is a $\beta$ such that $P^{x}\left(T_{\beta}^{(m)}<\infty\right)=0$ for all $m$, the proposition follows.

7. The multiplicative functional-when the processes have no holding points. In this section we assume $H=H^{*}=\varnothing$, i.e., both processes have no holding points. For $t \in[0, \infty)$ let $\Phi_{t}=\{\omega \in \Omega \mid$ for every $\delta>0$ there exists $(m, \alpha)$ such that $t \leqq T_{\alpha}^{(m)}(\omega)<t+\delta$ or $\left.X(t, \omega)=\Delta\right\}$. We now extend $M(\cdot, \omega)$ from $J(\omega)$ to $[0, \infty]$ by setting

$$
\begin{aligned}
M(t, \omega) & =\sup \left\{M\left(T_{\alpha}^{(m)}(\omega), \omega\right) \mid t \leqq T_{\alpha}^{(m)}(\omega)\right\} & & \text { if } \omega \in \Phi_{t}, \\
& =\inf \left\{M\left(T_{\alpha}^{(m)}(\omega), \omega\right) \mid t>T_{\alpha}^{(m)}(\omega)\right\} & & \text { if } \omega \in \Omega-\Phi_{t} .
\end{aligned}
$$

Lemma 6.2 guarantees that this is indeed an extension of the original $M(\cdot, \omega)$ for every $\omega . M$ is now a function on $[0, \infty] \times \Omega$. We will denote by $M(T)$ (or $\left.M_{T}\right)$, for any $T: \Omega \rightarrow[0, \infty]$, the function defined by $M(T)(\omega)=M(T(\omega), \omega)$. Note that 
$0 \leqq M \leqq 1$ and that $M(t, \omega)$ is nonincreasing in $t$ for every $\omega$. Moreover, if $M(\cdot, \omega)$ is right continuous on $J(\omega)$ then it is so on $[0, \infty]$. Hence Proposition 6.6 implies that $M(t)$ is right continuous in $t$ a.e.

Proposition 7.1. $M(t)$ is measurable over $\mathscr{F}_{t}$ for $0 \leqq t<\infty$.

Proof. We show first that $\Phi_{t} \in \mathscr{F}_{t}$. For an arbitrary $\mu$ let $\beta$ be such that $P^{\mu}\left\{T_{\beta}^{(m)}<\infty\right\}=0$ for all $m$. Then

$$
\Phi_{t}=\{X(t)=\Delta\} \cup\left(\bigcap_{k=1}^{\infty} \bigcup_{m=1}^{\infty} \bigcup_{\alpha<\beta}\left\{t \leqq T_{\alpha}^{(m)}<t+1 / k\right\}\right) \cup \Gamma,
$$

where $\Gamma$ is a subset of $\bigcup_{m=1}^{\infty}\left\{T_{\beta}^{(m)}<\infty\right\}$, a $P^{u}$-null set in $\mathscr{F}$. Since

$$
\bigcap_{k=1}^{\infty} \bigcup_{m=1}^{\infty} \bigcup_{\alpha<\beta}\left\{t \leqq T_{\alpha}^{(m)}<t+1 / k\right\} \in \bigcap_{k=1}^{\infty} \mathscr{F}(t+1 / k)=\mathscr{F}(t),
$$

the arbitrariness of $\mu$ implies $\Phi_{t} \in \mathscr{F}_{t}$. Now define a sequence of random variables $R_{n}$ as follows:

$$
\begin{aligned}
R_{n} & =\inf \left\{T_{\gamma}^{(n)} \mid T_{\gamma}^{(n)} \geqq t\right\} & & \text { on } \Phi_{t}, \\
& =\sup \left\{T_{\gamma}^{(n)} \mid T_{\gamma}^{(n)}<t\right\} & & \text { on } \Omega-\Phi_{t}
\end{aligned}
$$

Note that inf and sup above can be replaced by min and max respectively. Clearly as $n \rightarrow \infty, R_{n} \downarrow t$ on $\Phi_{t}-\{X(t)=\Delta\}$ and $R_{n} \uparrow \sup \left\{T_{\alpha}^{(m)} \mid T_{\alpha}^{(m)}<t\right\}$ on $\Omega-\Phi_{t}$. It then follows that $M\left(R_{n}\right) \rightarrow M(t)$ as $n \rightarrow \infty$. Now for a positive integer $k$ let $M^{(k)}\left(R_{n}\right)=M\left(R_{n}\right) I_{\left\{R_{n}<t+1 / k\right\}}$. Then $M^{(k)}\left(R_{n}\right) \rightarrow M(t)$ as $n \rightarrow \infty$. We will show that $M^{(k)}\left(R_{n}\right)$ is $\mathscr{F}(t+1 / k)$-measurable. This would imply that $M(t)$ is $\mathscr{F}(t+1 / k)$ measurable for every $k$ and thus $\mathscr{F}(t)$-measurable. To show $M^{(k)}\left(R_{n}\right)$ is measurable over $\mathscr{F}(t+1 / k)$, again let $\mu$ be arbitrary and choose $\beta$ such that $P^{\mu}\left(T_{\beta}^{(n)}<\infty\right)=0$ for every $n$. For each pair $(n, \gamma)$ let $\Phi_{t}(n, \gamma)=\Phi_{t} \cap\left\{T_{\gamma}^{(n)} \geqq t ; T_{\delta}^{(n)}<t\right.$ for all $\left.\delta<\gamma\right\}$ and $\Psi_{t}(n, \gamma)=\left\{T_{\gamma}^{(n)}<t \leqq T_{\gamma+1}^{(n)}\right\}-\Phi_{t}$. Clearly $\Phi_{t}(n, \gamma)$ and $\Psi_{t}(n, \gamma)$ are in $\mathscr{F}(t)$, and $R_{n}=T_{\gamma}^{(n)}$ on $\Phi_{t}(n, \gamma) \cup \Psi_{t}(n, \gamma)$. Since $M\left(T_{\gamma}^{(n)}\right)$ is $\mathscr{F}\left(T_{\gamma}^{(n)}\right)$-measurable, this implies that $M\left(R_{n}\right) I_{\Phi_{t}(n, \gamma) \cap\left(T_{y}^{(n)}<t+1 / k\right\}}$ and $M\left(R_{n}\right) I_{\Psi_{t}(n, \gamma)}$ are $\mathscr{F}(t+1 / k)$-measurable. Thus the function

$$
\sum_{\gamma<\beta}\left(M\left(R_{n}\right) I_{\Phi_{t}(n, \gamma) \cap\left\{T_{\gamma}^{(n)}<t+1 / k\right\}}+M\left(R_{n}\right) I_{\Psi_{t}(n, \gamma)}\right)
$$

is $\mathscr{F}(t+1 / k)$-measurable. Since this function differs from $M^{(k)}\left(R_{n}\right)$ only on a subset of $\Omega-\bigcup_{\gamma<\beta}\left(\Phi_{t}(n, \gamma) \cup \Psi_{t}(n, \gamma)\right) \subset\left\{T_{\beta}^{(n)}<\infty\right\}, M^{(k)}\left(R_{n}\right)$ is measurable over $\mathscr{F}(t+1 / k)^{\mu} . \mu$ being arbitrary, $M^{(k)}\left(R_{n}\right)$ is measurable over $\mathscr{F}(t+1 / k)$. The proof is thus complete.

We will now show that the restriction of $M$ to $[0, \infty) \times \Omega$, which we will still call $M$, is a multiplicative functional of $X$ and has the desired property. The assumption $H=\varnothing$ implies that for every $x$ and $t, P^{x}\left(\{X(t) \in E\}-\Phi_{t}\right)=0$. This fact will be strongly used in the next proof.

THEOREM 7.2. $M$ is a multiplicative functional of $X$. 
Proof. We have seen that $0 \leqq M \leqq 1, M(t)$ is nonincreasing and right continuous in $t$ a.e., and $M(t)$ is $\mathscr{F}(t)$-measurable. It remains to show that for arbitrary $s, t \geqq 0$ the equality

$$
M(s+t)=M(t)\left[M(s)\left(\theta_{t}\right)\right]
$$

holds a.e. Obviously we need only to show the equality holding a.e. on $\{s+t<\sigma\}$. Since $\{s+t<\sigma\}=\{X(s+t) \in E\}=\{X(s+t) \in E\} \cap\{X(t) \in E\}$,

$P^{x}\left(\{s+t<\sigma\}-\Phi_{t} \cap \Phi_{s+t}\right) \leqq P^{x}\left(\{X(t) \in E\}-\Phi_{t}\right)+P^{x}\left(\{X(s+t) \in E\}-\Phi_{s+t}\right)=0$.

Thus it suffices to show that (7.1) holds a.e. on $\Phi_{s} \cap \Phi_{s+t} \cap\{s+t<\sigma\}$.

For each $n$ define $R_{n}$ as in the proof of Proposition 7.1, and define $R_{n}^{\prime}$ as $R_{n}$ except that $t$ is replaced by $s+t$, i.e., $R_{n}^{\prime}=\inf \left\{T_{y}^{(n)} \mid T_{y}^{(n)} \geqq s+t\right\}$ on $\Phi_{s+t}$ and $=\sup \left\{T_{\gamma}^{(n)} \mid T_{\gamma}^{(n)}<s+t\right\}$ elsewhere. On $\Phi_{t} \cap \Phi_{s+t}, R_{n} \leqq R_{n}^{\prime}$, although both may be infinite. Let $S_{n}=R_{n}^{\prime}-R_{n}$ on $\left\{R_{n}<\infty\right\}$. Then

$$
M\left(R_{n}^{\prime}\right)=M\left(R_{n}\right)\left[M\left(S_{n}\right)\left(\theta_{R_{n}}\right)\right]
$$

a.e. on $\left\{R_{n}<\infty\right\} \cap \Phi_{t} \cap \Phi_{s+t}\left(M\left(S_{n}\right)\left(\theta_{R_{n}}\right)(\omega)=M\left(S_{n}(\omega), \theta_{R_{n}} \omega\right)\right)$. This follows easily from Lemma 6.4. Now $M\left(R_{n}\right) \rightarrow M(t)$ and $M\left(R_{n}^{\prime}\right) \rightarrow M(s+t)$ as $n \rightarrow \infty$. Since $\left\{R_{n}<\infty, t<\sigma\right\} \uparrow\{t<\sigma\}$ we need only to show

$$
M\left(S_{n}\right)\left(\theta_{R_{n}}\right) \rightarrow M(s)\left(\theta_{t}\right) \quad \text { as } n \rightarrow \infty
$$

a.e. on $\Phi_{t} \cap \Phi_{s+t} \cap\{s+t<\sigma\}$ (regard $M\left(S_{n}\right)\left(\theta_{R_{n}}\right)$ as zero if $R_{n}=\infty$ ). In view of Corollary 2.4, for each $n$ and $\omega \in \Phi_{t} \cap \Phi_{s+t}$ there exist $\delta_{n}(\omega) \leqq \delta_{n}^{\prime}(\omega) \leqq \pi$ such that $R_{n}(\omega)=t+T_{\delta_{n}(\omega)}^{(n+1)}\left(\theta_{t} \omega\right)$ and $R_{n}^{\prime}(\omega)=t+T_{\delta_{n}^{\prime}(\omega)}^{(n+1)}\left(\theta_{t} \omega\right)$. For a fixed $x$ choose $\beta$ such that $P^{x}\left(T_{\beta}^{(n+2)}<\infty\right)=0$. It is easy to see that if $T_{\beta}^{(n+2)}(\omega)=\infty$ then $\delta_{n}(\omega)$ and $\delta_{n}^{\prime}(\omega)$ can be chosen $\leqq \beta$. Now from Lemma 6.4 and the Markov property we have

$$
M\left(T_{\gamma^{\prime}}^{(n+1)}\right)\left(\theta_{t}\right)=\left[M\left(T_{\gamma}^{(n+1)}\right)\left(\theta_{t}\right)\right]\left[M\left(T_{\gamma^{\prime \prime}}^{(n+1}\right)\left(\theta_{T_{\gamma}^{(n+1)}}\right)\left(\theta_{t}\right)\right]
$$

a.e. $P^{x}$ on $\left\{T_{\gamma}^{(n+1)}\left(\theta_{t}\right)<\infty\right\}$ whenever $\gamma+\gamma^{\prime \prime}=\gamma^{\prime}$. It follows that

$$
M\left(T_{\delta_{n}^{\prime}}^{(n+1)}\right)\left(\theta_{t}\right)=\left[M\left(T_{\delta_{n}}^{(n+1)}\right)\left(\theta_{t}\right)\right]\left[M\left(T_{\delta_{n}^{\prime \prime}}^{(n+1)}\right)\left(\theta_{\left.T_{\delta}^{(n+1)}\right)}\left(\theta_{t}\right)\right]\right.
$$

a.e. $P^{x}$ on $\left\{R_{n} \leqq \infty\right\} \cap \Phi_{t} \cap \Phi_{s+t}$, where $\delta_{n}^{\prime \prime}(\omega)$ is defined by $\delta_{n}(\omega)+\delta_{n}^{\prime \prime}(\omega)=\delta_{n}^{\prime}(\omega)$.

Now the second factor on the right side of (7.3) is exactly $M\left(S_{n}\right)\left(\theta_{R_{n}}\right)$. It is clear that $T_{\delta_{n}}^{(n+1)}\left(\theta_{t}\right)=R_{n}-t \downarrow 0$ and $T_{\delta_{n}^{\prime}}^{(n+1)}\left(\theta_{t}\right)=R_{n}-t \downarrow s$ on $\Phi_{t} \cap \Phi_{s+t} \cap\{s+t<\sigma\}$. Since by the Markov property $M\left(t^{\prime}\right)\left(\theta_{t}\right)$ is right continuous in $t^{\prime}$ a.e. $P^{x}$, $M\left(T_{\delta_{n}}^{(n+1)}\right)\left(\theta_{t}\right) \rightarrow 1$ and $M\left(T_{\delta_{n}^{\prime}}^{(n+1)}\right)\left(\theta_{t}\right) \rightarrow M(s)\left(\theta_{t}\right)$ a.e. $P^{x}$ on $\Phi_{t} \cap \Phi_{s+t} \cap\{s+t<\sigma\}$ as $n \rightarrow \infty$. Thus (7.2) follows from (7.3), and this completes the proof of (7.1) and hence of the theorem.

LEMma 7.3. Let $K$ be a compact subset of E. For each $m$ let $R_{m}=\inf \left\{T_{\alpha}^{(m)} \mid T_{\alpha}^{(m)} \geqq T_{K}\right\}$. If $x \notin K$, then $E^{x}\left\{M\left(R_{m}\right) ; X\left(R_{m}\right) \in B, R_{m}<\infty\right\}={ }^{*} P^{x}\left(X\left(R_{m}\right) \in B, R_{m}<\infty\right)$ for all $B \in \mathscr{B}_{\Delta}$. 
Proof. Let $\beta$ be such that $P^{x}\left(T_{\beta}^{(m)}<\infty\right)={ }^{*} P^{x}\left(T_{\beta}^{(m)}<\infty\right)=0$. Then for $B \in \mathscr{B}_{\Delta}$ $E^{x}\left\{M\left(R_{n}\right) ; X\left(R_{m}\right) \in B, R_{m}<\infty\right\}=\sum_{\alpha<\beta} E^{x}\left\{M\left(T_{\alpha}^{(m)}\right) ; X\left(T_{\alpha}^{(m)}\right) \in B, R_{m}=T_{\alpha}^{(m)}<\infty\right\}$ and

$$
{ }^{*} P^{x}\left(X\left(R_{m}\right) \in B, R_{m}<\infty\right)=\sum_{\alpha<\beta} * P^{x}\left(X\left(T_{\alpha}^{(m)}\right) \in B, R_{m}=T_{\alpha}^{(m)}<\infty\right) .
$$

Now it follows from Lemma 3.4 that $\left\{X\left(T_{\alpha}^{(m)}\right) \in B, R_{m}=T_{\alpha}^{(m)}<\infty\right\} \in \mathscr{H}_{\infty}(m, \alpha)^{\varepsilon_{x}}$. Since obviously $R_{m}<\sigma$ if $R_{m}<\infty$, we have

$$
E^{x}\left\{M\left(T_{\alpha}^{(m)}\right) ; X\left(T_{\alpha}^{(m)}\right) \in B, R_{m}=T_{\alpha}^{(m)}<\infty\right\}={ }^{*} P^{x}\left(X\left(T_{\alpha}^{(m)}\right) \in B, R_{m}=T_{\alpha}^{(m)}<\infty\right)
$$

by Corollary 4.7. The desired equality follows.

THEOREM 7.4. For every $x \in E$, compact subset $K$ of $E$, and $B \in \mathscr{B}$

$$
E^{x}\left\{M\left(T_{K}\right) ; X\left(T_{K}\right) \in B, T_{K}<\infty\right\}=H_{K}^{*}(x, B) .
$$

Proof. (i) Suppose first $x \notin K$. Define $R_{m}$ as in the previous proof. We have shown in Proposition 2.6 that $R_{m} \downarrow T_{K}$ a.e. $P^{x}$ and a.e. ${ }^{*} P^{x}$. It then follows from the a.e. right continuity of $M(t)$ and the previous lemma that, for $f \in \mathscr{C}_{0}$,

$$
\begin{aligned}
{ }^{*} E^{x}\left\{f\left(X\left(T_{K}\right)\right) ; T_{K}<\infty\right\} & =\lim _{m}^{*} E^{x}\left\{f\left(X\left(R_{m}\right)\right) ; R_{m}<\infty\right\} \\
& =\lim _{m} E^{x}\left\{M\left(R_{m}\right) f\left(X\left(R_{m}\right)\right) ; R_{m}<\infty\right\} \\
& =E^{x}\left\{M\left(T_{K}\right) f\left(X\left(T_{K}\right)\right) ; T_{K}<\infty\right\} .
\end{aligned}
$$

The arbitrariness of $f$ implies (7.4) for all $B \in \mathscr{B}$.

(ii) Suppose now $x \in K$. By Proposition $1.2 x \in \operatorname{reg} K$ if and only if $x \in \mathrm{reg}^{*} K$. Hence if $x \in \operatorname{reg} K, T_{K}=0$ a.e. $P^{x}$ and a.e. ${ }^{*} P^{x}$, and it follows that

$$
\begin{aligned}
E^{x}\left\{M\left(T_{K}\right) ; X\left(T_{K}\right) \in B, T_{K}<\infty\right\}={ }^{*} P^{x}\left(X\left(T_{K}\right) \in B, T_{K}<\infty\right)=1 & \text { if } x \in B, \\
=0 & \text { if } x \notin B .
\end{aligned}
$$

If $x \notin \operatorname{reg} K$, then $x \notin \operatorname{reg*} K$. Let $F_{n}=\{y \mid \rho(x, \Delta) / 2 \geqq \rho(x, y) \geqq 1 / n\}$. We have $T_{F_{n}} \downarrow 0$ a.e. $P^{x}$ and a.e. ${ }^{*} P^{x}$. Hence $P^{x}\left(T_{F_{n}}<T_{K}\right) \uparrow 1$ and ${ }^{*} P^{x}\left(T_{F_{n}}<T_{K}\right) \uparrow 1$ as $n \rightarrow \infty$. Now it follows from the fact established in (i) that, for all $n$,

$$
\begin{aligned}
& { }^{*} E^{x}\left\{{ }^{*} P^{X\left(T_{F_{n}}\right)}\left(X\left(T_{K}\right) \in B, T_{K}<\infty\right) ; X\left(T_{F_{n}}\right) \notin K, T_{F_{n}}<\infty\right\} \\
& \quad=E^{x}\left\{M\left(T_{F_{n}}\right) E^{X\left(T_{F_{n}}\right)}\left[M\left(T_{K}\right) ; X\left(T_{K}\right) \in B, T_{K}<\infty\right] ; X\left(T_{F_{n}}\right) \notin K, T_{K}<\infty\right\} .
\end{aligned}
$$

Clearly the left side converges to ${ }^{*} P^{x}\left(X\left(T_{K}\right) \in B, T_{K}<\infty\right)$ as $n \rightarrow \infty$. Since on the set $\left\{F_{n}<T_{K}\right\}, M\left(T_{K}\right)=M\left(T_{F_{n}}\right)\left[M\left(T_{K}\right)\left(\theta_{T_{F_{n}}}\right)\right]$ a.e. $P^{x}$, the right side must converge to $E^{x}\left\{M\left(T_{K}\right) ; X\left(T_{K}\right) \in B, T_{K}<\infty\right\}$ as $n \rightarrow \infty$. Hence (7.4) follows, and the proof is complete.

8. The multiplicative functional-when the processes have holding points. If $H=H^{*}$ is nonempty, the functional defined in the previous section does not have 
the desired property (in fact it is not necessarily a multiplicative functional), and we have to make a different definition. Let $R=\inf _{m} T_{1}^{(m)}=\inf \{t<\sigma \mid X(t) \neq X(0)\}$. For each $t$ set $M(t)=\sup \left\{M\left(T_{\alpha}^{(n)}\right) \mid T_{\alpha}^{(n)} \geqq t\right\}$ on $\Phi_{t}$. Note that $M\left(t+R\left(\theta_{t}\right)\right)$ is defined for every $t$. We then set on $\Omega-\Phi_{t}$

$$
\begin{aligned}
M(t)= & \min \left\{M\left(t+R\left(\theta_{t}\right)\right) / M(R)\left(\theta_{t}\right), \inf \left\{M\left(T_{\alpha}^{(n)}\right) \mid T_{\alpha}^{(n)}<t\right\}\right\} \\
& \quad \text { if } \sup \left\{T_{\alpha}^{(n)} \mid T_{\alpha}^{(n)}<t\right\} \neq T_{\gamma}^{(m)} \text { for any }(m, \gamma), \\
= & \inf \left\{M\left(T_{\alpha}^{(n)}\right) \mid T_{\alpha}^{(n)}<t\right\} \quad \text { otherwise. }
\end{aligned}
$$

We will show that this $M$ (restricted to $[0, \infty) \times \Omega$ ) is a desired multiplicative functional. It is obvious that $M(t)$ is nonincreasing in $t$ everywhere. If $M(\cdot, \omega)$ is right continuous on $J(\omega)$ then it is so on $[0, \infty]$. Hence $M(t)$ is right continuous a.e.

For a while let $K$ be a fixed compact subset of $H$. Define stopping times $R_{\gamma}$ and $S_{\gamma}, \gamma<\pi$, inductively as follows: $R_{0}=S_{0}=0 ; S_{\gamma+1}=R_{\gamma}+T_{K}\left(\theta_{R_{\gamma}}\right)$ if $R_{\gamma}<\infty$ and $=\infty$ if otherwise; $R_{\gamma}=S_{\gamma}+R\left(\theta_{S_{\gamma}}\right)$ if $S_{\gamma}<\infty$ and $=\infty$ if otherwise; $S_{\gamma}=\sup _{\delta<\gamma} R_{\delta}$ $=\sup _{\delta<\gamma} S_{\delta}$ for a limit ordinal $\gamma$. Obviously, $X\left(S_{\gamma}\right) \in K$ a.e. and a.e. ${ }^{*} ; R_{\gamma}>S_{\gamma}$ a.e. and a.e. ${ }^{*}$ on $\left\{S_{\gamma}<\infty\right\}$ for $\gamma>0$, so for each $\mu$ there exists $\beta$ such that $P^{\mu}\left(R_{\beta}<\infty\right)$ $={ }^{*} P^{\mu}\left(R_{\beta}<\infty\right)=0$; for each $\mu, R_{\gamma}=$ some $T_{\alpha}^{(m)}$ ( $m$ and $\alpha$ depending on $\left.\omega\right)$ a.e. and a.e. ${ }^{*}$. For simplicity we assume that for each $\gamma$ and $\omega R_{\gamma}(\omega)=T_{\alpha}^{(m)}(\omega)$ for some $(m, \alpha)$.

Let $T_{n}=\inf \left\{T_{\alpha}^{(n)}<\sigma \mid\right.$ if $X\left(T_{\alpha}^{(n)}\right) \in V_{i}^{(n)}$ then $\left.K \cap U_{i}^{(n)} \neq \varnothing\right\}$. Let $\mathscr{I}_{n}$ be the $\sigma$-field generated by sets of the form $\left\{X\left(T_{\alpha}^{(m)}\right) \in B, T_{\alpha}^{(m)} \leqq T_{n}, T_{\alpha}^{(m)}<\sigma\right\}$ or $\left\{X\left(S_{1}\right) \in B, S_{1}<\sigma\right\}$. Let $\mathscr{I}_{\infty}$ be the smallest $\sigma$-field containing all $\mathscr{I}_{n}$. Let $\mathscr{K}$ be the $\sigma$-field generated by sets of the form $\left\{X\left(T_{\alpha}^{(m)}\right) \in B, T_{\alpha}^{(m)} \leqq R_{1}, T_{\alpha}^{(m)}<\sigma\right\}$. Let $\gamma$ be an arbitrary limit ordinal and let $\gamma_{1}<\cdots<\gamma_{n} \uparrow \gamma$. Let $\mathscr{J}_{n}$ be the $\sigma$-field generated by sets of the form $\left\{X\left(T_{\alpha}^{(m)}\right) \in B, T_{\alpha}^{(m)} \leqq R_{\gamma_{n}}, T_{\alpha}^{(m)}<\sigma\right\}$ or $\left\{X\left(S_{\gamma}\right) \in B, S_{\gamma}<\infty\right\}$. Let $\mathscr{J}_{\infty}$ be the smallest $\sigma$-field containing all $\mathscr{J}_{n}$. Let $\mathscr{K}_{\gamma}$ be the $\sigma$-field generated by sets of the form $\left\{X\left(T_{\alpha}^{(m)}\right) \in B, T_{\alpha}^{(m)} \leqq R_{\gamma}, T_{\alpha}^{(m)}<\sigma\right\}$. We will omit the proofs of the following two lemmas.

LEMMA 8.1. (i) For each $\mu, \mathscr{I}_{1}^{\mu} \subset \ldots \subset \mathscr{I}_{n}^{\mu} \subset \ldots \subset \mathscr{I}_{\infty}^{\mu} \subset \mathscr{K}^{\mu}$; (ii) for each $\mu M\left(T_{n}\right)$ is $\mathscr{I}_{n}^{\mu}$-measurable; (iii) for every $x$ and $\Lambda \in \mathscr{I}_{n} \cap\left\{T_{n}<\sigma\right\},{ }^{*} P^{x}(\Lambda)=E^{x}\left\{M\left(T_{n}\right) ; \Lambda\right\}$; (iv) $X\left(T_{n}\right)$ is $\mathscr{I}_{n}^{\mu}$-measurable for each $\mu$.

LEMMA 8.2. (i) For each $\mu, \mathscr{J}_{1}^{\mu} \subset \ldots \subset \mathscr{J}_{n}^{\mu} \subset \ldots \subset \mathscr{J}_{\infty}^{\mu} \subset \mathscr{K}_{\gamma}^{\mu}$; (ii) for each $\mu$ $M\left(R_{\gamma_{n}}\right)$ is $\mathscr{J}_{n}^{\mu}$-measurable; (iii) for each $x$ and $\Lambda \in \mathscr{J}_{n} \cap\left\{R_{\gamma_{n}}<\sigma\right\},{ }^{*} P^{x}(\Lambda)$ $=E^{x}\left\{M\left(R_{\gamma_{n}}\right) ; \Lambda\right\}$; (iv) $X\left(R_{\gamma_{n}}\right)$ is $\mathscr{J}_{n}^{\mu}$-measurable for each $\mu$.

Let $h: E \times E \rightarrow[0,1]$ be $\mathscr{B} \times \mathscr{B}$-measurable and satisfy

$$
H_{K}^{*}(x, B)=\int_{B} h(x, y) H_{K}(x, d y)
$$

for $x \in E$ and $B \in \mathscr{B}$. Let $\phi_{n}=h\left(X\left(T_{n}\right), X\left(S_{1}\right)\right)$ on $\left\{S_{1}<\sigma\right\}$ and $=0$ elsewhere.

LEMMA 8.3. For each $x,\left\{\phi_{n} M\left(T_{n}\right), n=1,2, \ldots\right\}$ is a martingale with respect to the o-fields $\mathscr{I}_{n}^{\varepsilon}, n=1,2, \ldots$, and the measure $P^{x}$. 
Proof. One shows that for $\Lambda \in \mathscr{I}_{n} \cap\left\{S_{1}<\sigma\right\},{ }^{*} P^{x}(\Lambda)=E^{x}\left\{\phi_{n} M\left(T_{n}\right) ; \Lambda\right\}$.

Let $N\left(S_{1}\right)=\lim \inf _{n} \phi_{n} M\left(T_{n}\right)$. Then we have

Corollary 8.4. For each $x$ and $\Lambda \in \mathscr{K} \cap\left\{S_{1}<\sigma\right\}, E^{x}\left\{N\left(S_{1}\right) ; \Lambda\right\}={ }^{*} P^{x}(\Lambda)$.

LEMMA 8.5. (i) ${ }^{*} P^{x}(X(R) \in B, R<\sigma)=E^{x}\{M(R) ; X(R) \in B, R<\sigma\}$ for every $x$ and $B \in \mathscr{B}$; (ii) $M\left(R_{1}\right)=N\left(S_{1}\right)\left[M(R)\left(\theta_{S_{1}}\right)\right]$ a.e. on $\left\{S_{1}<\infty\right\}$.

Proof. (i) is easy to see. Both sides of (ii) are $\mathscr{K}^{\varepsilon} \varepsilon_{x}$-measurable, and it follows from (i) and Corollary 8.4 that for $\Lambda \in \mathscr{K}, E^{x}\left\{M\left(R_{1}\right) ; \Lambda\right\}=E^{x}\left\{N\left(S_{1}\right)\left[M(R)\left(\theta_{S_{1}}\right)\right] ; \Lambda\right\}$ $={ }^{*} P^{x}\left(\Lambda \cap\left\{R_{1}<\sigma\right\}\right)$.

Let $T=\inf \{t \mid X(t) \neq X(0)\}$ and for each $p$ let $A_{p}=\left\{y \in K \mid E^{y}(T)>1 / p\right\}$. Then $A_{p} \uparrow K$ as $p \rightarrow \infty$. From Lemma 8.2 we obtain for $B \in \mathscr{B}$

$$
{ }^{*} E^{x}\left\{H_{A_{P}}^{*}\left(X\left(R_{\gamma_{n}}\right), B\right) ; R_{y_{n}}<\sigma\right\} \leqq E^{x}\left\{M\left(R_{y_{n}}\right) H_{A_{p}}\left(X\left(R_{\gamma_{n}}\right), B\right) ; R_{\gamma_{n}}<\sigma\right\} .
$$

It is not hard to see that ${ }^{*} P^{x}\left(X\left(R_{\delta}\right) \in A_{p}\right.$ for some $\left.\delta, \gamma_{n} \leqq \delta<\gamma\right) \rightarrow 0$ and $P^{x}\left(X\left(R_{\delta}\right) \in A_{p}\right.$ for some $\left.\delta, \gamma_{n} \leqq \delta<\gamma\right) \rightarrow 0$ as $n \rightarrow \infty$. Hence as $n \rightarrow \infty$ we obtain from the above inequality

$$
\begin{aligned}
& * P^{x}\left(X\left(S_{y}\right) \in A_{p} \cap B, S_{\gamma}<\sigma\right)+{ }^{*} E^{x}\left\{H_{A_{p}}^{*}\left(X\left(S_{\gamma}\right), B\right) ; X\left(S_{y}\right) \notin A_{p}, S_{\gamma}<\sigma\right\} \\
& \leqq E^{x}\left\{\lim _{n} M\left(R_{\gamma_{n}}\right) ; X\left(S_{\gamma}\right) \in A_{p} \cap B, S_{\gamma}<\sigma\right\} \\
&+E^{x}\left\{\lim _{n} M\left(R_{\gamma_{n}}\right) H_{A_{p}}\left(X\left(S_{y}\right), B\right) ; X\left(S_{\gamma}\right) \notin A_{p}, S_{\gamma}<\sigma\right\} .
\end{aligned}
$$

As $p \rightarrow \infty$ we obtain

$$
{ }^{*} P^{x}\left(X\left(S_{\gamma}\right) \in K \cap B, S_{\gamma}<\sigma\right) \leqq E^{x}\left\{\lim _{n} M\left(R_{\gamma_{n}}\right) ; X\left(S_{\gamma}\right) \in K \cap B, S_{\gamma}<\sigma\right\}
$$

Thus we have

Lemma 8.6. For every $x$ and $B \in \mathscr{B}$,

$$
{ }^{*} P^{x}\left(X\left(S_{\gamma}\right) \in B, S_{\gamma}<\sigma\right) \leqq E^{x}\left\{\lim _{n} M\left(R_{\gamma_{n}}\right) ; X\left(S_{\gamma}\right) \in B, S_{y}<\sigma\right\} .
$$

It is not hard to show that both sides of the above inequality are $\mathscr{B}_{\Delta}$-measurable in $x$. Hence there exists $c_{y}: E \times E \rightarrow[0,1], \mathscr{B} \times \mathscr{B}$-measurable, such that

$$
{ }^{*} P^{x}\left(X\left(S_{y}\right) \in B, S_{\gamma}<\sigma\right)=E^{x}\left\{c_{\gamma}\left(X(0), X\left(S_{\gamma}\right)\right) \lim _{n} M\left(R_{\gamma_{n}}\right) ; X\left(S_{y}\right) \in B, S_{\gamma}<\sigma\right\}
$$

for all $x \in E$ and $B \in \mathscr{B}$. Let $\delta_{n}$ be such that $\gamma_{n}+\delta_{n}=\gamma$ and assume that $c_{\delta_{n}}$ are defined. Let $\psi_{n}=c_{\delta_{n}}\left(X\left(R_{\gamma_{n}}\right), X\left(R_{\gamma}\right)\right)$ on $\left\{R_{\gamma}<\sigma\right\}$ and $=0$ elsewhere.

LEMMA 8.7. For each $x,\left\{\psi_{n}, n=1,2, \ldots\right\}$ is a martingale with respect to $\left\{\mathscr{J}_{n}^{\varepsilon} x, n=1,2, \ldots\right\}$ and the measure $\lim _{m} M\left(R_{\gamma_{m}}\right) d P^{x}$.

Proof. Applying Lemma 8.2 one shows that for $\Lambda \in \mathscr{J}_{n} \cap\left\{S_{\gamma}<\sigma\right\}$,

$$
{ }^{*} P^{x}(\Lambda)=E^{x}\left\{\psi_{n} \lim _{m} M\left(R_{\gamma_{m}}\right) ; \Lambda\right\}
$$


Let $\psi=\lim \inf _{n} \psi_{n}$. Let $N\left(S_{\gamma}\right)=\psi \lim _{m} M\left(R_{\gamma_{m}}\right)$. Then we have

Corollary 8.8. For $\Lambda \in \mathscr{J}_{\infty} \cap\left\{S_{\gamma}<\sigma\right\}, E^{x}\left\{N\left(S_{\gamma}\right) ; \Lambda\right\}={ }^{*} P^{x}(\Lambda)$.

LEMMA 8.9. $M\left(R_{\gamma}\right)=N\left(S_{\gamma}\right)\left[M(R)\left(\theta_{S_{\gamma}}\right)\right]$ a.e. on $\left\{S_{\gamma}<\infty\right\}$.

Proof. Both sides are $\mathscr{K}_{y}^{\varepsilon} x$-measurable for each $x$. One shows that, for $\Lambda \in \mathscr{K}_{y}$, $E^{x}\left\{M\left(R_{y}\right) ; \Lambda\right\}=E^{x}\left\{N\left(S_{y}\right)\left[M(R)\left(\theta_{S_{y}}\right)\right] ; \Lambda\right\}={ }^{*} P^{x}\left(\Lambda \cap\left\{R_{y}<\sigma\right\}\right)$.

For each $\gamma$ let $N\left(S_{\gamma+1}\right)=M\left(R_{\gamma}\right)\left[N\left(S_{1}\right)\left(\theta_{R_{\gamma}}\right)\right]$ on $\left\{R_{\gamma}<\sigma\right\}$ and $=0$ elsewhere. Then it follows from Lemma 8.5 that $M\left(R_{\gamma+1}\right)=N\left(S_{\gamma+1}\right)\left[M(R)\left(\theta_{S_{y+1}}\right)\right]$ a.e. on $\left\{S_{\gamma+1}<\infty\right\}$.

LEMMA 8.10. For every $\gamma, M(t)=N\left(S_{\gamma}\right)$ a.e. on $\left\{S_{\gamma} \leqq t<R_{\gamma}\right\}$.

Proof. This is easily deduced from Lemmas 8.5 and 8.9 , the remark preceding the lemma, and the definition of $M(t)$ (note that $M(R)\left(\theta_{t}\right)=M(R)\left(\theta_{S_{y}}\right)$ on $\left.\left\{S_{\gamma} \leqq t<R_{\gamma}\right\}\right)$.

Proposition 8.11. $M(t)$ is $\mathscr{F}(t)$-measurable for every $t$.

Proof. According to the proof of Proposition $7.1 M(t) I_{\left(X(t) \in E_{\left.\Delta_{0}-H\right)}\right.}$ is $\mathscr{F}(t)$ measurable. For a given $\mu$ there exist increasing compact subsets $K_{n}$ of $H$ such that $P^{\mu}\left(X(t) \in K_{n}\right) \uparrow P^{\mu}(X(t) \in H)$. It follows that to prove $M(t) I_{\{X(t) \in H\}}$ is $\mathscr{F}(t)$ measurable it suffices to show $M(t) I_{\{X(t) \in H\}}$ is $\mathscr{F}(t)$-measurable for all compact subsets $K$ of $H$. Construct $N\left(S_{\gamma}\right)$ as before. Since $N\left(S_{\gamma}\right)$ is clearly $\mathscr{F}\left(S_{y}\right)$-measurable it follows from Lemma 8.10 that $M(t) I_{\left\{S_{y} \leqq t<R_{y}\right\}}$ is $\mathscr{F}(t)$-measurable. This implies $M(t) I_{\{X(t) \in \dot{H}\}}$ is $\mathscr{F}(t)$-measurable since for a fixed $\mu$ there exists $\beta$ such that $P^{\mu}\left(R_{\beta}<\infty\right)=0$.

Proposition 8.12. For every $s$ and $t, M(s+t)=M(t)\left[M(s)\left(\theta_{t}\right)\right]$ a.e.

Proof. We need only to show that the equality holds a.e. on $\{X(t) \in K$ or $X(s+t) \in K\}, K$ a compact subset of $H$. And it suffices to prove the equality holding a.e. on

$$
\begin{aligned}
& \left.\Lambda_{1}=\left\{S_{y} \leqq t<R_{\gamma}, S_{\gamma^{\prime}} \leqq s+t<R_{\gamma^{\prime}}\right\} \quad \text { (we may assume } \gamma<\gamma^{\prime}\right), \\
& \Lambda_{2}=\left\{S_{y} \leqq t<R_{y}, X(s+t) \in I\right\}, \text { and } \Lambda_{3}=\left\{X(t) \in I, S_{y} \leqq s+t<R_{\gamma}\right\} .
\end{aligned}
$$

We show only $M(s+t)=M(t)\left[M(s)\left(\theta_{t}\right)\right]$ a.e. on $\Lambda_{1}$ as the rest follows from similar arguments together with the proof of Theorem 7.2. Suppose $\gamma+\delta=\gamma^{\prime}$. Then

$$
\begin{aligned}
M(s+t) & =N\left(S_{\gamma^{\prime}}\right)=M\left(R_{\gamma}\right)\left[N\left(S_{\delta}\right)\left(\theta_{R_{\gamma}}\right)\right]=N\left(S_{y}\right)\left[M(R)\left(\theta_{S_{y}}\right)\right]\left[N\left(S_{\delta}\right)\left(\theta_{R_{\gamma}}\right)\right] \\
& =N\left(S_{\gamma}\right)\left[N\left(S_{\delta}\right)\left(\theta_{S_{\delta}}\right)\right]=M(t)\left[M(s)\left(\theta_{t}\right)\right] \text { a.e. }
\end{aligned}
$$

These equalities are not hard to see. For the last equality note that $M(t)$ depends only on the trajectory of the path.

Thus we have proved that $M$ is a multiplicative functional of $X$. The following theorem will establish its desired property.

THEOREM 8.13. For $x \in E$ and compact $K \subset E, E^{x}\left\{M\left(T_{K}\right) ; X\left(T_{K}\right) \in B, T_{K}<\infty\right\}$ $=H_{K}^{*}(x, B)$ for all $B \in \mathscr{B}$. 
Proof. If $K \subset I$, the theorem follows from the proof of Theorem 7.4. If $K \subset H$, it is a result of Corollary 8.4 and the fact that $M\left(T_{K}\right)=M\left(S_{1}\right)=N\left(S_{1}\right)$ a.e. In general we find compact $K_{m} \subset K \cap H$ and compact $K_{m}^{\prime} \subset K \cap I$ such that $T_{K_{m}} \downarrow T_{K \cap H}$ and $T_{K_{m}^{\prime}} \downarrow T_{K \cap I}$ a.e. $P^{x}$ and a.e. ${ }^{*} P^{x}$ so that $T_{K_{m} \cup K_{m}^{\prime}} \downarrow T_{K}$ a.e. $P^{x}$ and a.e. ${ }^{*} P^{x}$ as $m \rightarrow \infty$. It then suffices to show

$$
E^{x}\left\{M\left(T_{K_{m} \cup K_{m}^{\prime}}\right) ; X\left(T_{K_{m} \cup K_{m}^{\prime}}\right) \in B, T_{K_{m} \cup K_{m}^{\prime}}<\infty\right\}=H_{K_{m} \cup K_{m}^{\prime}}^{*}(x, B)
$$

for all $B$. Let $T_{n}=\inf \left\{T_{\alpha}^{(n)}<\sigma \mid X\left(T_{\alpha}^{(n)}\right) \in V_{i}^{(n)} \Rightarrow U_{i}^{(n)} \cap\left(K_{m} \cup K_{m}^{\prime}\right) \neq \varnothing\right\}$. Let $N$ be so large that $\rho\left(K_{m}, K_{m}^{\prime}\right)=\inf \left\{\rho(y, z) \mid y \in K_{m}, z \in K_{m}^{\prime}\right\}>1 / 2 N$, and let

$$
F=\left\{y \in E \mid \rho\left(y, K_{m}\right) \leqq 1 / N\right\} \text { and } F^{\prime}=\left\{y \in E \mid \rho\left(y, K_{m}^{\prime}\right) \leqq 1 / N\right\} .
$$

If $n \geqq N$, then we have

$$
\begin{aligned}
{ }^{*} E^{x}\left\{H_{K_{m}}^{*}\left(X\left(T_{n}\right), B\right) ; X\left(T_{n}\right) \in F, T_{n}<\sigma\right\} \\
\quad+{ }^{x}\left\{H_{K_{m}^{\prime}}^{*}\left(X\left(T_{n}\right), B\right) ; X\left(T_{n}\right) \in F^{\prime}, T_{n}<\sigma\right\} \\
=E^{x}\left\{M\left(T_{n}\right) E^{X\left(T_{n}\right)}\left\{M\left(T_{K_{m}}\right) ; X\left(T_{K_{m}}\right) \in B, T_{K_{m}}<\infty\right\} ; X\left(T_{n}\right) \in F, T_{n}<\sigma\right\} \\
\quad+E^{x}\left\{M\left(T_{n}\right) E^{X\left(T_{n}\right)}\left\{M\left(T_{K_{m}^{\prime}}\right) ; X\left(T_{K_{m}^{\prime}}\right) \in B, T_{K_{m}^{\prime}}<\infty\right\} ; X\left(T_{n}\right) \in F^{\prime}, T_{n}<\sigma\right\} .
\end{aligned}
$$

Now it follows from the quasi-left continuity that $P^{x}\left(T_{K_{m}}<T_{K_{m}^{\prime}}, X\left(T_{n}\right) \in F\right) \rightarrow 1$ and $P^{x}\left(T_{K_{m}^{\prime}}<T_{K_{m}}, X\left(T_{n}\right) \in F^{\prime}\right) \rightarrow 1$ as $n \rightarrow \infty$, with similar convergences holding when ${ }^{*} P^{x}$ replaces $P^{x}$. Applying the bounded convergence theorem we obtain (8.1) from (8.2). Thus the theorem is proved.

\section{REFERENCES}

1. R. M. Blumenthal, R. K. Getoor and H. P. McKean, Jr., Markov processes with identical hitting distributions, Illinois J. Math. 6 (1962), 402-420.

2. E. B. Dynkin, Markov processes (English transl.), Springer-Verlag, Berlin, 1965.

3. R. K. Getoor, Additive functions of a Markov process, Lecture notes, Univ. of Hamburg, 1964.

4. - Additive functionals and excessive functions, Ann. Math. Statist. 36 (1965), 409-422.

5. P. A. Meyer, Fonctionnelles multiplicatives et additives de Markov, Ann. Inst. Fourier (Grenoble) 12 (1962), 125-213.

6. M. G. Sur, A class of Markov processes whose exit probabilities are majorized by the exit probabilities of a Wiener process, Dokl. Akad. Nauk SSSR 147 (1962), 323-326 = Soviet Math. Dokl. 3 (1962), 1626-1629.

$$
\begin{aligned}
& \text { UNIVERSITY OF WASHINGTON, } \\
& \text { Seattle, Washington } \\
& \text { Cornell University, } \\
& \text { ITHACA, New York }
\end{aligned}
$$

